\title{
Polyphenolic Compounds as Pancreatic Lipase Inhibitors
}

Authors

Affiliation
Tina Buchholz, Matthias F. Melzig

Institute of Pharmacy, Freie Universität Berlin, Berlin, Germany
Key words

- fat-digestion

- anti-obesity

- pancreatic lipase inhibition

- polyphenols

\section{Abstract \\ $\nabla$}

Obesity and its associated diseases such as diabetes mellitus and coronary heart diseases are a major challenge for our society. An important target for the treatment of obesity includes the development of inhibitors of nutrient digestion and absorption. Inhibition of pancreatic lipase and the associated reduction of lipid absorption is an attractive approach for the discovery of potent agents. Currently, the only clinically approved pharmacologic agent as pancreatic lipase inhibitor is Orlistat. However, its usage is compromised by unpleasant gastrointestinal adverse reactions (oily stools, oily spotting, flatulence). The use of botanical materials as a potential source of new

\section{Introduction}

$\nabla$

Overweight and obesity are globally the fifth leading risks for death. At least 2.8 million adults die each year as a result of being overweight or obese [1]. Worldwide obesity is so common that it is beginning to replace undernutrition and infectious diseases as the most significant contributor to ill health. Frequently, the risks associated with obesity are diabetes mellitus, coronary heart disease, certain forms of cancer and sleep-breathing disorders $[2,3]$.

An important target for the treatment of obesity includes the development of inhibitors of nutrient digestion and absorption [4]. Inhibition of pancreatic lipase (PL) and the associated reduction of lipid absorption is an attractive approach for the discovery of potent agents to the treatment of obesity [5-7]. To find new compounds with PL inhibitory activity for anti-obesity agents several plant, bacterial, fungal and marine species have been screened [8-10]. Currently, Orlistat is the only pharmacologic agent as pancreatic lipase inhibitor clinically approved in Europe. Orlistat is drugs is of increasing importance and application. Natural products that are interesting for obesity treatment are generally considered to have less toxic and side effects than totally synthetic drugs. One of the most important sources of potential pancreatic lipase inhibitors represents the class of polyphenols. This article summarizes most studied subclasses of polyphenols including flavonoids, hydroxycinnamic acids, hydroxybenzoic acids and lignans with pancreatic lipase inhibitory effects. A structural comparison of potent inhibitors shows an increased inhibitory effect depending on number and position of phenolic hydroxyl groups, degree of polymerization and elimination of glycosylation during digestion. is directed towards identifying novel inhibitors that lack some of these adverse reactions during patient treatment. The use of plant based resources as a potential platform for discovery and development of new drugs has become a lucrative research field in this context. One advantage of natural products is their presence in high concentrations in the gut after oral administration, because no further metabolic processes are necessary for an effect. Furthermore, systemic adverse effects are reduced by low absorption rates. Natural products generally have the advantage in being mild in their activity, in having a broad spectrum of possible multiple/synergetic effects and are considered to have less toxic and side effects than those construed on a totally synthetic based drug designing $[14,15]$. Possible adverse 


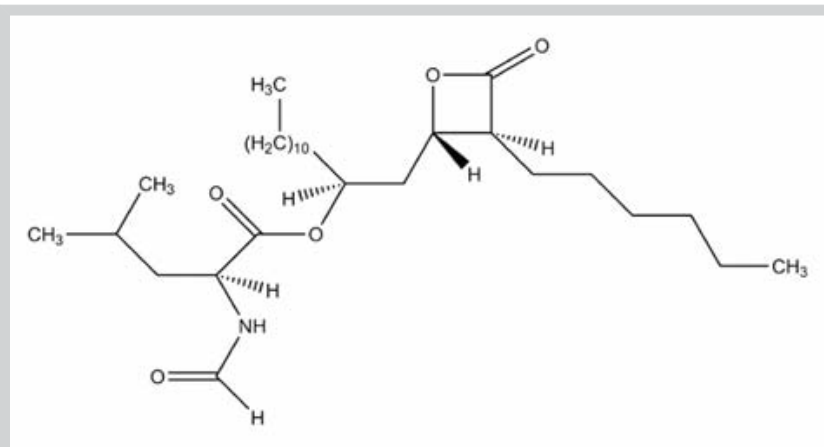

Fig. 1 Chemical structure of tetrahydrolipstatin (common name: Orlistat).

reactions of the natural products are rather unclear because metabolites resulting from microbial degradation in the human body are largely unknown. Some available data on the metabolic products of the ellagitannins (Urolitine) show a positive effect in the treatment of breast cancer by inhibition of proliferation of the affected cells [16]. Known negative side effects of polyphenols are the toxic effect of tannins to microorganisms and high concentrations of green tea polyphenols have been reported to cause liver and kidney damage in animal studies $[17,18]$. However, these side effects were observed at high concentrations and for a clear understanding of potential adverse effects of dietary polyphenols further studies are necessary.

\section{Obesity}

$\nabla$

Obesity is a medical condition caused by an imbalance between energy intake and expenditure. It is defined as abnormal or excess body fat accumulation to the extent that it may have an adverse effect on health [19]. Healthy body fat percentages vary depending on age, gender and body type. The normal amount of body fat is between $25-30 \%$ for women and $18-23 \%$ for men. Women are considered obese with a body fat percentage over $30 \%$, men with over $25 \%$ [20]. A crude population measure of obesity is the body mass index (BMI). It is defined as the individual's body mass in $\mathrm{kg}$ divided by the square of height in $\mathrm{m} \mathrm{[20].}$ According to WHO, an adult with a BMI equal to or more than 25 is considered overweight and is considered obese with a BMI of 30 or higher. Obesity can be divided into three categories: Grade I obesity: BMI 25-29.9, grade II obesity: BMI 30-39.9, and grade III obesity: BMI > 40 [15]. An elevated BMI value is a major risk factor for diseases such as cardiovascular diseases (mainly heart diseases and stroke), some cancers (endometrial, breast, and colon) and diabetes mellitus [21]. For a long time obesity was not recognized as an illness, but it certainly represents a typical example of diseases evolving from the advancement of civilization, being strongly related to lifestyle. In 2013, obesity was classified as a disease by the American Medical Association [22].

\section{Lipid Metabolism and Pancreatic Lipase} $\nabla$

Ingested fats are metabolized and absorbed in the intestine. They play important roles in energy supply, thermal regulation, and energy storage and as typical membrane components. Some lip- ids or associated fractions also function as transmission/reservoir agents for essential fatty acids and fat-soluble vitamins [23,24]. About $95 \%$ of the ingested fat can be absorbed by the human intestine. The main fat constituents (90\%) of a typical Western diet are triacylglycerides (TAGs). A TAG is an ester derived from a single molecule of glycerol and three fatty acids. Since TAGs cannot be absorbed, they must be hydrolyzed. The triacylglycerides are split via intestinal enzymes into monoacylglycerol and free fatty acids and can be absorbed by the duodenum [23]. Human pancreatic lipase [HPL, pancreatic triacylglycerol lipase (EC: 3.1 .1 .3 )] is the main enzyme that breaks down dietary fats in the human digestive system. It is produced in the glandular cells of the pancreas and secreted into the duodenum $[25,26]$. The HPL is a glycoprotein with a molecular weight of $52 \mathrm{kDa}$ and consists of a 16 amino acid signal peptide and a mature protein of 449 amino acids [25]. The polypeptide chain is divided into two folding units, the $\mathrm{N}$-terminal domain (amino acid residues 1-336) and the $\mathrm{C}$ terminal domain (amino acid residues 337-449). While the Cterminal domain is responsible for the binding of the co-lipase, the $\mathrm{N}$-terminal domain is catalytically active $[25,27]$. The enzyme has a catalytic triad of the amino acids aspartic acid (176), histidine (263) and serine (152) in its active center that represents the lipolytic site. Serine (152) is essential for the hydrolytic activity of the enzyme. For the full functioning of the enzyme, the cofactor co-lipase is required [27]. The co-lipase is a non-enzymatic protein of $10 \mathrm{kDa}$ and consists of 100 amino acids. It is secreted as a pro-form and is converted by tryptic cleavage of an N-terminal pentapeptide (enterostatin) in the much more active form [28]. The entering of the chyme in the duodenum induces the secretion of various hormones of the intestinal tract (cholecystokinin, secretin). These cause the secretion of bile salts and pancreatic enzymes in the intestine [29]. HPL hydrolyzes $40 \%$ to $70 \%$ of TAGs [27]. Short-chain fatty acids (< C12) enter the portal vein and are transported directly into the liver. Free fatty acids and monoacylglyceroles, which were absorbed by the intestinal mucosa cells, are re-esterified to TAGs. These are incorporated into chylomicrons and reach the lymphatic vessels [30]. Enzyme levels and activity (especially lipases), physiochemical properties of dietary lipids and the presence of inhibitors or enhancers are the major determinants that can affect the lipolysis rates [23].

\section{Plant Products as Inhibitors of Pancreatic Lipase $\nabla$}

One screening strategy used in discovery of anti-obesity drugs is the search of potent lipase inhibitors from plant extracts. A variety of natural bioactive compounds present in plants have been screened for their potential effect on metabolic diseases including obesity. In particular, the class of secondary metabolites showed a high potential for inhibitors [27]. Inter alia, the inhibitory effect of tannins on proteases was detected in numerous studies [31-33]. They are able to form insoluble/soluble complexes with proteins due to nonspecific binding to the surface of the protein [31]. This makes them a good target for the treatment of obesity by PL inhibition. However, more research is needed for identification and characterization of effective lipase inhibitors. Crude plant extracts do not only contain active components but also non-active components. Possible multiple and synergetic effects of the active components complicate their identification and characterization. Also, the inhibitory mechanisms (reversible/irreversible) of plant products on PL remain unclear. It was generally attributed to the ability of secondary metabolites, e.g. 
tannins, to bind, complex and precipitate protein although some studies reported non-competitive inhibition mechanisms [34]. A second and much more innovative approach lies in using the structure of a known potent inhibitor, determining the binding site and pharmacore using in-silico computational modelling experiments and deriving the most likely potential natural structures (hits) using the available plant resources data banks and consequently testing these under experimental conditions to confirm/validate their activity. This review summarizes the most studied chemical classes of polyphenols with pancreatic lipase inhibitory activity including flavonoids, hydroxycinnamic acids, hydroxybenzoic acids and lignans. It is specialized in works where either pure polyphenols or crude plant extracts with a correlation to contained polyphenols have been tested for possible inhibition of PL. The most significant results up to October 2014 are presented.

\section{Determination of pancreatic lipase activity}

To determine the inhibitory potential of the natural compounds different in vitro enzyme assays are available. In most cases, fluorescence or absorption-based detection methods are used [3538]. The most commonly used fluorescent assay is based on the hydrolysis of an oleate ester of 4-methylumbelliferone (4-MUO) [36,38-41]. Under the presence of an active enzyme the highly fluorescent cleavage product 4-methylumbelliferone (4-MU) is released. The accompanying increase in fluorescence is proportional to lipase activity. The use of fluorescence-based assays has two decisive advantages: they are more sensitive than absorption-based assays and also turbid solutions are measurable. However, their use is often associated with higher costs. A commonly used in vitro absorption assay for the determination of PL activity is measuring the release of oleic acid from triolein [36]. Furthermore, various esters of $p$-nitrophenol (acetate, butyrate, octanoate) are used as substrates $[35,37,42]$. The released $p$-nitrophenol can be measured photometrically. Another typical lipase substrate for the photometric determination is 2,3-dimercapto-1-propanol tributyrate [43]. In addition to experimental work, a computer-based analysis is also possible. Jeong et al. used a surface response methodology for the assessment of the inhibitory potential [44]. In general, the synthetic product Orlistat was used as a positive control.

\section{Polyphenols}

In a variety of studies the beneficial effect of polyphenols on lipid metabolism could be demonstrated. They inhibit enzymes including PL, lipoprotein lipase (LPL), and glycerophosphate dehydrogenase (GPDH) [45]. Polyphenols containing extracts are able to lower body weight, fat weight, plasma free fatty acid levels, and hepatic lipid accumulation [46]. In the following, the current research results of the most studied subgroups of polyphenols for PL inhibitory activity are shown.

\section{Flavonoids}

Flavonoids represent the most common group of polyphenolic compounds in the human diet and are found ubiquitously in plants with kaempferol and quercetin as the main aglycon representatives $[47,48]$. They protect the plant from harmful external influences, therefore the highest concentrations are found in the outer leaves or shells. Flavonoids consist of two aromatic carbon rings, benzopyran (A and $C$ rings), and benzene ( $B$ ring) ( $O$ Fig. 2A) [47]. They can be divided into different subgroups: chalcones, dihydrochalcones, aurones, flavones, flavonols, dihy-

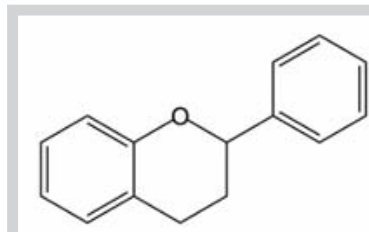

A

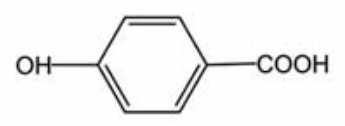

C

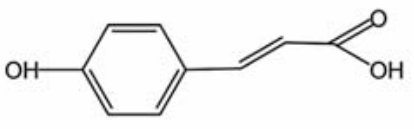

B

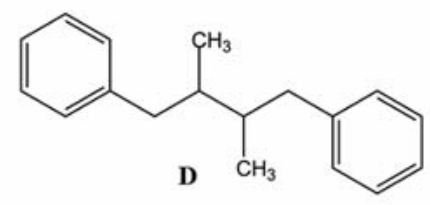

Fig. 2 Basic structure of A flavonoids (2-phenylchromane), B hydroxycinnamic acids, C hydroxybenzoic acids, D dibenzylbutane (lignan).

droflavonoles, flavanones, flavanols, anthocyanidins, leucoanthocyanidins, proanthocyanidins, biflavonoids and isoflavonoids [49]. This classification is based on the degree of the oxidation of the C-ring, the hydroxylation pattern of the ring structure and the substitution of the C3-position [47]. Foods with a high flavonoid content are onions, legumes, broccoli, berries and orange juice [50].

Identified flavonoids with inhibitory potential are shown in $\odot$ Table 1. In the continued search for effective agents against obesity and metabolic syndrome, several plant species have been screened to find active compounds to inhibit PL. In the following proven structure-effect relationships and other features of the identified active components are explained in more detail:

Isolated flavonoid compounds from Intsia palembanica Miq. (Leguminosae) were analyzed by Batubara et al. [43]. The tested substances were (-)-robidanol, (+)-epirobidanol, 4'-dehydroxyrobidanol, fustin, ampelopsin, naringenin, robinetin, myricetin, quercetin and 3,7,3',5'- tetrahydroxyflavone. Lipase inhibitory activity assay was conducted using the 2,3-dimercapto-1-propanol tributyrate (BALB) method (in vitro enzyme assay). Seven of ten tested compounds showed a lipase inhibitory effect in a dose-dependent manner ( $\mathrm{IC}_{50}$ : 13.7-835.0 $\left.\mu \mathrm{M}\right)$. (+)-epirobidanol, robinetin and naringenin showed an activation of lipase activity [43]. However, Assini et al. reported in further studies, that citrus flavonoids such as naringenin, hersperidin, nobiletin, and taugeretin showed a promising therapeutic effect for the treatment of metabolic dysregulation. In experimental and clinical studies lipidlowering, insulin-sensitizing, antihypertensive and anti-inflammatory effects could be confirmed [51].

C-glycoside flavones from methanolic extracts of the leaves of Eremochloa ophiuroides (Munro) Hack. (Poaceae) showed an inhibitory potential. $\mathrm{IC}_{50}$ values were determined using $p$-nitrophenyl-butyrate as substrate (in vitro) and ranged from 18.5$50.5 \mu \mathrm{M}$. It was possible to estimate the optimal position and number of glycosyl groups on the flavone skeleton for pancreatic lipase inhibitory activity. The highest inhibitory effect showed $C$ glycosyl flavones with two sugar moieties at C6 [35].

Lychee (Litchi chinensis Sonn., Sapindaceae) flower-water extracts (LFWEs) were tested on lipase inhibition by measuring the rate of releasing of oleic acid from triolein (in vitro). The extracts contained phenolic acids, flavonoids, condensed tannins, anthocyanins, and proanthocyanidins. The following flavonoids could be identified in the extract: (+)-catechin, (-)-epicatechin, 
Table 1 Comparison of active flavonoids with PL inhibitory activity from different sources. $I C_{50}$ is indicated when available.

\begin{tabular}{|c|c|c|c|c|c|c|}
\hline Active compound & Source & Family & $\begin{array}{l}\mathrm{IC}_{50} \text { (active } \\
\text { compound) }\end{array}$ & $\mathrm{IC}_{50}$ (extract) & Test system & Reference(s) \\
\hline (-) Epicatechin & $\begin{array}{l}\text { Litchi chinensis } \\
\text { Sonn. }\end{array}$ & Sapindaceae & - & $\begin{array}{l}\mathrm{IC}_{44.69}: 7 \mathrm{mg} / \mathrm{mL} \\
\text { (water extract) }\end{array}$ & $\begin{array}{l}\text { release of oleic acid } \\
\text { from triolein (in vitro) }\end{array}$ & [36] \\
\hline $\begin{array}{l}\text { (-)-Catechin 3-O-gal- } \\
\text { late }\end{array}$ & $\begin{array}{l}\text { Camellia sinensis } \\
\text { (L.) Kuntze }\end{array}$ & Theaceae & $0.543 \mu \mathrm{M}$ & - & $\begin{array}{l}\text { release of 4-MU from } \\
\text { 4-MUO (in vitro) }\end{array}$ & [39] \\
\hline $\begin{array}{l}\text { (-)-Epiafzelechin 3-O- } \\
\text { gallate }\end{array}$ & $\begin{array}{l}\text { Camellia sinensis } \\
\text { (L.) Kuntze }\end{array}$ & Theaceae & $2.582 \mu \mathrm{M}$ & - & $\begin{array}{l}\text { release of 4-MU from } \\
\text { 4-MUO (in vitro) }\end{array}$ & [39] \\
\hline $\begin{array}{l}\text { (-)-Epicatechin }(4 \beta-8) \\
\text { (-)-epigallocatechin } 3 \text { - } \\
\text { O-gallate }\end{array}$ & $\begin{array}{l}\text { Camellia sinensis } \\
\text { (L.) Kuntze }\end{array}$ & Theaceae & $0.147 \mu \mathrm{M}$ & - & $\begin{array}{l}\text { release of 4-MU from } \\
\text { 4-MUO (in vitro) }\end{array}$ & [39] \\
\hline $\begin{array}{l}\text { (-)-Epicatechin 3-O-(3'- } \\
\text { O-methyl)gallate }\end{array}$ & $\begin{array}{l}\text { Camellia sinensis } \\
\text { (L.) Kuntze }\end{array}$ & Theaceae & $0.680 \mu \mathrm{M}$ & - & $\begin{array}{l}\text { release of 4-MU from } \\
\text { 4-MUO (in vitro) }\end{array}$ & [39] \\
\hline \multirow[t]{3}{*}{$\begin{array}{l}\text { (-)-Epicatechin 3-O- } \\
\text { gallate }\end{array}$} & $\begin{array}{l}\text { Camellia sinensis } \\
\text { (L.) Kuntze }\end{array}$ & Theaceae & $0.452 \mu \mathrm{M}$ & - & $\begin{array}{l}\text { release of 4-MU from } \\
\text { 4-MUO (in vitro) }\end{array}$ & [39] \\
\hline & $\begin{array}{l}\text { Camellia sinensis } \\
\text { (L.) Kuntze }\end{array}$ & Theaceae & $2.37 \mu \mathrm{M}$ & - & $\begin{array}{l}\text { release of 4-MU from } \\
\text { 4-MUO (in vitro) }\end{array}$ & {$[40]$} \\
\hline & - & - & $13 \mu \mathrm{M}$ & - & $\begin{array}{l}\text { release of 4-MU from } \\
\text { 4-MUO (in vitro) }\end{array}$ & [63] \\
\hline $\begin{array}{l}\text { (-)-Epicatechin 3-O- } \\
\text { gallate }(4 \beta-8)-(-) \text {-epi- } \\
\text { gallocatechin 3-O-gal- } \\
\text { late }\end{array}$ & $\begin{array}{l}\text { Camellia sinensis } \\
\text { (L.) Kuntze }\end{array}$ & Theaceae & $0.846 \mu \mathrm{M}$ & - & $\begin{array}{l}\text { release of 4-MU from } \\
\text { 4-MUO (in vitro) }\end{array}$ & [39] \\
\hline (-)-Epigallocatechin & $\begin{array}{l}\text { Camellia sinensis } \\
\text { (L.) Kuntze }\end{array}$ & Theaceae & $128 \mu \mathrm{M}$ & - & $\begin{array}{l}\text { release of 4-MU from } \\
\text { 4-MUO (in vitro) }\end{array}$ & {$[40]$} \\
\hline $\begin{array}{l}\text { (-)-Epigallocatechin } \\
(4 \beta-8)-(-) \text {-epicatechin } \\
\text { 3-O-gallate }\end{array}$ & $\begin{array}{l}\text { Camellia sinensis } \\
\text { (L.) Kuntze }\end{array}$ & Theaceae & $0.913 \mu \mathrm{M}$ & - & $\begin{array}{l}\text { release of 4-MU from } \\
\text { 4-MUO (in vitro) }\end{array}$ & [39] \\
\hline $\begin{array}{l}\text { (-)-Epigallocatechin } \\
\text { 3,5-di-O-gallate }\end{array}$ & $\begin{array}{l}\text { Camellia sinensis } \\
\text { (L.) Kuntze }\end{array}$ & Theaceae & $0.098 \mu \mathrm{M}$ & - & $\begin{array}{l}\text { release of 4-MU from } \\
\text { 4-MUO (in vitro) }\end{array}$ & [39] \\
\hline \multirow[t]{5}{*}{$\begin{array}{l}\text { (-)-Epigallocatechin 3- } \\
\text { O-gallate }\end{array}$} & $\begin{array}{l}\text { Camellia sinensis } \\
\text { (L.) Kuntze }\end{array}$ & Theaceae & $0.349 \mu \mathrm{M}$ & - & $\begin{array}{l}\text { release of 4-MU from } \\
\text { 4-MUO (in vitro) }\end{array}$ & [39] \\
\hline & $\begin{array}{l}\text { Camellia sinensis } \\
\text { (L.) Kuntze }\end{array}$ & Theaceae & $0.177 \mu \mathrm{M}$ & - & $\begin{array}{l}\text { release of 4-MU from } \\
\text { 4-MUO (in vitro) }\end{array}$ & {$[40]$} \\
\hline & $\begin{array}{l}\text { Ligularia fischeri } \\
\text { (Ledeb.) Turcz. }\end{array}$ & Compositae & $1.8 \mu \mathrm{M}$ & - & $\begin{array}{l}\text { release of 4-MU from } \\
\text { 4-MUO (in vitro) }\end{array}$ & [41] \\
\hline & - & - & $11 \mu \mathrm{M}$ & - & $\begin{array}{l}\text { release of 4-MU from } \\
\text { 4-MUO (in vitro) }\end{array}$ & {$[63]$} \\
\hline & - & - & $0.8 \mu \mathrm{M}$ & - & $\begin{array}{l}\text { release of 4-MU from } \\
\text { 4-MUO (in vitro) }\end{array}$ & [34] \\
\hline 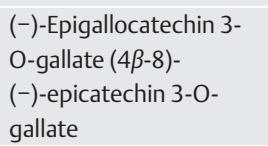 & $\begin{array}{l}\text { Camellia sinensis } \\
\text { (L.) Kuntze }\end{array}$ & Theaceae & $0.612 \mu \mathrm{M}$ & - & $\begin{array}{l}\text { release of 4-MU from } \\
\text { 4-MUO (in vitro) }\end{array}$ & [39] \\
\hline $\begin{array}{l}\text { (-)-Epigallocatechin 3- } \\
\text { O-p-coumaroate }\end{array}$ & $\begin{array}{l}\text { Camellia sinensis } \\
\text { (L.) Kuntze }\end{array}$ & Theaceae & $0.885 \mu \mathrm{M}$ & - & $\begin{array}{l}\text { release of 4-MU from } \\
\text { 4-MUO (in vitro) }\end{array}$ & [39] \\
\hline $\begin{array}{l}\text { (-)-Gallocatechin 3,5- } \\
\text { di-O-gallate }\end{array}$ & $\begin{array}{l}\text { Camellia sinensis } \\
\text { (L.) Kuntze }\end{array}$ & Theaceae & $0.213 \mu \mathrm{M}$ & - & $\begin{array}{l}\text { release of 4-MU from } \\
\text { 4-MUO (in vitro) }\end{array}$ & [39] \\
\hline $\begin{array}{l}\text { (-)-Gallocatechin 3-O- } \\
\text { gallate }\end{array}$ & $\begin{array}{l}\text { Camellia sinensis } \\
\text { (L.) Kuntze }\end{array}$ & Theaceae & $0.437 \mu \mathrm{M}$ & - & $\begin{array}{l}\text { release of 4-MU from } \\
\text { 4-MUO (in vitro) }\end{array}$ & [39] \\
\hline (-)-Robidanol & $\begin{array}{l}\text { Intsia palembanica } \\
\text { Miq. }\end{array}$ & Leguminosae & $100.2 \mu \mathrm{M}$ & $\begin{array}{l}4.10 \mu \mathrm{g} / \mathrm{mL}(\mathrm{MeOH} \\
\text { extract) }\end{array}$ & $\begin{array}{l}\text { hydrolysis of 2,3-dimer- } \\
\text { capto-1-propanol tribu- } \\
\text { tyrate (in vitro) }\end{array}$ & {$[43]$} \\
\hline \multirow[t]{3}{*}{$(+)$ Catechin } & $\begin{array}{l}\text { Litchi chinensis } \\
\text { Sonn. }\end{array}$ & Sapindaceae & - & $\begin{array}{l}\mathrm{IC}_{44.69}: 7 \mathrm{mg} / \mathrm{mL} \\
\text { (water extract) }\end{array}$ & $\begin{array}{l}\text { release of oleic acid } \\
\text { from triolein (in vitro) }\end{array}$ & [36] \\
\hline & Mentha aquatica L. & Lamiaceae & - & - & $\begin{array}{l}\text { hydrolysis of 4-nitro- } \\
\text { phenyl octanoate } \\
\text { (in vitro) }\end{array}$ & [37] \\
\hline & $\begin{array}{l}\text { Vitis rotundifolia } \\
\text { Michx. }\end{array}$ & Vitaceae & $3.42 \mathrm{mM}$ & $\begin{array}{l}8.63 \mathrm{mg} / \mathrm{mL} \\
(80 \%(\mathrm{v} / \mathrm{v}) \mathrm{MeOH} \\
\text { extract) }\end{array}$ & $\begin{array}{l}\text { release of 4-MU from } \\
\text { 4-MUO (in vitro) }\end{array}$ & [64] \\
\hline $\begin{array}{l}\text { (+)-Catechin (4R-8)- } \\
\text { (-)-epigallocatechin }\end{array}$ & $\begin{array}{l}\text { Camellia sinensis } \\
\text { (L.) Kuntze }\end{array}$ & Theaceae & $7.912 \mu \mathrm{M}$ & - & $\begin{array}{l}\text { release of 4-MU from } \\
\text { 4-MUO (in vitro) }\end{array}$ & [39] \\
\hline $\begin{array}{l}\text { (+)-Catechin (4R-8)- } \\
\text { (-)-epigallocatechin }\end{array}$ & $\begin{array}{l}\text { Camellia sinensis } \\
\text { (L.) Kuntze }\end{array}$ & Theaceae & $0.174 \mu \mathrm{M}$ & - & $\begin{array}{l}\text { release of 4-MU from } \\
\text { 4-MUO (in vitro) }\end{array}$ & [39] \\
\hline
\end{tabular}


Table 1 Continued

\begin{tabular}{|c|c|c|c|c|c|c|}
\hline Active compound & Source & Family & $\begin{array}{l}\mathrm{IC}_{50} \text { (active } \\
\text { compound) }\end{array}$ & $\mathrm{IC}_{50}$ (extract) & Test system & Reference(s) \\
\hline $\begin{array}{l}(+) \text { Gallocatechin } \\
(4 R-8)-(-) \text {-epicatechin }\end{array}$ & $\begin{array}{l}\text { Camellia sinensis } \\
\text { (L.) Kuntze }\end{array}$ & Theaceae & $2.862 \mu \mathrm{M}$ & - & $\begin{array}{l}\text { release of 4-MU from } \\
\text { 4-MUO (in vitro) }\end{array}$ & [39] \\
\hline $\begin{array}{l}3,7,3^{\prime}, 5^{\prime} \text {-Tetrahydroxy- } \\
\text { flavone }\end{array}$ & $\begin{array}{l}\text { Intsia palembanica } \\
\text { Miq. }\end{array}$ & Leguminosae & $835.0 \mu \mathrm{M}$ & $\begin{array}{l}4.10 \mu \mathrm{g} / \mathrm{mL} \\
\text { (MeOH extract) }\end{array}$ & $\begin{array}{l}\text { hydrolysis of 2,3-dimer- } \\
\text { capto-1-propanol tribu- } \\
\text { tyrate (in vitro) }\end{array}$ & [43] \\
\hline $\begin{array}{l}\text { 3-O-b-xylopyranosyl- } \\
(1 \rightarrow 2) \text {-O-b-galactopyr- } \\
\text { anoside }\end{array}$ & $\begin{array}{l}\text { Filipendula camt- } \\
\text { schatica (Pall.) } \\
\text { Maxim. }\end{array}$ & Rosaceae & $300.00 \mu \mathrm{M}$ & - & $\begin{array}{l}\text { release of 4-MU from } \\
\text { 4-MUO (in vitro) }\end{array}$ & [65] \\
\hline 4'-Dehydroxyrobidanol & $\begin{array}{l}\text { Intsia palembanica } \\
\text { Miq. }\end{array}$ & Leguminosae & $40.0 \mu \mathrm{M}$ & $\begin{array}{l}4.10 \mu \mathrm{g} / \mathrm{mL} \\
\text { (MeOH extract) }\end{array}$ & $\begin{array}{l}\text { hydrolysis of 2,3-dimer- } \\
\text { capto-1-propanol tribu- } \\
\text { tyrate (in vitro) }\end{array}$ & [43] \\
\hline $\begin{array}{l}\text { 5,7,4'-Trihydroxy-6,8- } \\
\text { diprenylisoflavone }\end{array}$ & $\begin{array}{l}\text { Cudrania tricuspi- } \\
\text { data (Carrière) } \\
\text { Bureau ex Lavallée }\end{array}$ & Moraceae & $65.0 \mu \mathrm{M}$ & - & $\begin{array}{l}\text { response surface meth- } \\
\text { odology with three-lev- } \\
\text { el three-factor Box- } \\
\text { Behnken design (BBD) }\end{array}$ & [44] \\
\hline $\begin{array}{l}\text { 6-C- } \beta \text {-D-boivinopyrano- } \\
\text { side }\end{array}$ & $\begin{array}{l}\text { Eremochloa ophiur- } \\
\text { oides (Munro) } \\
\text { Hack. }\end{array}$ & Poaceae & $50.5 \mu \mathrm{M}$ & - & $\begin{array}{l}\text { hydrolysis of p-nitro- } \\
\text { phenyl-butyrate } \\
\text { (in vitro) }\end{array}$ & [35] \\
\hline $\begin{array}{l}\text { 8-C-ascorbyl(-)-epigal- } \\
\text { locatechin }\end{array}$ & $\begin{array}{l}\text { Camellia sinensis } \\
\text { (L.) Kuntze }\end{array}$ & Theaceae & $0.646 \mu \mathrm{M}$ & - & $\begin{array}{l}\text { release of 4-MU from } \\
\text { 4-MUO (in vitro) }\end{array}$ & [39] \\
\hline $\begin{array}{l}\text { 8-C-ascorbyl(-)-epigal- } \\
\text { locatechin 3-O-gallate }\end{array}$ & $\begin{array}{l}\text { Camellia sinensis } \\
\text { (L.) Kuntze }\end{array}$ & Theaceae & $0.791 \mu \mathrm{M}$ & - & $\begin{array}{l}\text { release of 4-MU from } \\
\text { 4-MUO (in vitro) }\end{array}$ & [39] \\
\hline Ampelopsin & $\begin{array}{l}\text { Intsia palembanica } \\
\text { Miq. }\end{array}$ & Leguminosae & $36.0 \mu \mathrm{M}$ & $\begin{array}{l}4.10 \mu \mathrm{g} / \mathrm{mL} \\
\text { (MeOH extract) }\end{array}$ & $\begin{array}{l}\text { hydrolysis of 2,3-dimer- } \\
\text { capto-1-propanol tribu- } \\
\text { tyrate (in vitro) }\end{array}$ & [43] \\
\hline Cyanidin & - & - & $591.8 \mu \mathrm{M}$ & - & $\begin{array}{l}\text { release of 4-MU from } \\
\text { 4-MUO (in vitro) }\end{array}$ & [64] \\
\hline $\begin{array}{l}\text { Cyanidin-3,5-digluco- } \\
\text { side }\end{array}$ & - & - & $1.38 \mathrm{mM}$ & - & $\begin{array}{l}\text { release of 4-MU from } \\
\text { 4-MUO (in vitro) }\end{array}$ & [64] \\
\hline Cyanidin-3-glucoside & $\begin{array}{l}\text { Santalum acumina- } \\
\text { tum (R. Br.) A. DC. }\end{array}$ & Santalaceae & - & $\begin{array}{l}0.6 \mathrm{mg} / \mathrm{mL} \\
{[80 \%(\mathrm{v} / \mathrm{v}) \mathrm{MeOH}} \\
\text { extract }]\end{array}$ & $\begin{array}{l}\text { release of 4-MU from } \\
\text { 4-MUO (in vitro) }\end{array}$ & [66] \\
\hline Daidzein & - & - & $19 \mu \mathrm{M}$ & - & $\begin{array}{l}\text { release of 4-MU from } \\
\text { 4-MUO (in vitro) }\end{array}$ & [63] \\
\hline Delphinidin & $\begin{array}{l}\text { Vitis rotundifolia } \\
\text { Michx. }\end{array}$ & Vitaceae & - & $\begin{array}{l}11.15 \mathrm{mg} / \mathrm{mL} \\
{[80 \%(\mathrm{v} / \mathrm{v}) \mathrm{MeOH}} \\
\text { extract }]\end{array}$ & $\begin{array}{l}\text { release of 4-MU from } \\
\text { 4-MUO (in vitro) }\end{array}$ & [64] \\
\hline Derhamnosylmaysin & $\begin{array}{l}\text { Eremochloa } \\
\text { ophiuroides } \\
\text { (Munro) Hack. }\end{array}$ & Poaceae & $25.9 \mu \mathrm{M}$ & - & $\begin{array}{l}\text { hydrolysis of } p \text {-nitro- } \\
\text { phenyl-butyrate } \\
\text { (in vitro) }\end{array}$ & [35] \\
\hline Fustin & $\begin{array}{l}\text { Intsia palembanica } \\
\text { Miq. }\end{array}$ & Leguminosae & $13.7 \mu \mathrm{M}$ & $\begin{array}{l}4.10 \mu \mathrm{g} / \mathrm{mL} \\
\text { (MeOH extract) }\end{array}$ & $\begin{array}{l}\text { hydrolysis of 2,3-dimer- } \\
\text { capto-1-propanol tribu- } \\
\text { tyrate (in vitro) }\end{array}$ & [43] \\
\hline Galangin & $\begin{array}{l}\text { Alpinia galangal (L.) } \\
\text { Willd. }\end{array}$ & Zingiberaceae & $178.4 \mathrm{mM}$ & - & & [67] \\
\hline Genistein & - & - & $20 \mu \mathrm{M}$ & - & $\begin{array}{l}\text { release of 4-MU from } \\
\text { 4-MUO (in vitro) }\end{array}$ & [63] \\
\hline Hesperidin & $\begin{array}{l}\text { Citrus reticulata } \\
\text { Blanco }\end{array}$ & Rutaceae & $52.4 \mu \mathrm{M}$ & - & $\begin{array}{l}\text { release of 4-MU from } \\
\text { 4-MUO (in vitro) }\end{array}$ & [53] \\
\hline Hyperin & - & - & $44 \mu \mathrm{M}$ & - & $\begin{array}{l}\text { release of 4-MU from } \\
\text { 4-MUO (in vitro) }\end{array}$ & [63] \\
\hline Isoorientin & $\begin{array}{l}\text { Eremochloa } \\
\text { ophiuroides } \\
\text { (Munro) Hack. }\end{array}$ & Poaceae & $44.6 \mu \mathrm{M}$ & - & $\begin{array}{l}\text { hydrolysis of } p \text {-nitro- } \\
\text { phenyl-butyrate } \\
\text { (in vitro) }\end{array}$ & [35] \\
\hline $\begin{array}{l}\text { Isoorientin 2-O- } \alpha \text {-L- } \\
\text { rhamnoside }\end{array}$ & $\begin{array}{l}\text { Eremochloa } \\
\text { ophiuroides } \\
\text { (Munro) Hack. }\end{array}$ & Poaceae & $18.5 \mu \mathrm{M}$ & - & $\begin{array}{l}\text { hydrolysis of } p \text {-nitro- } \\
\text { phenyl-butyrate } \\
\text { (in vitro) }\end{array}$ & [35] \\
\hline Isoquercitrin & - & - & $25 \mu \mathrm{M}$ & - & $\begin{array}{l}\text { release of 4-MU from } \\
\text { 4-MUO (in vitro) }\end{array}$ & [63] \\
\hline \multirow[t]{3}{*}{ Kaempferol } & - & - & $2.5 \mu \mathrm{M}$ & - & $\begin{array}{l}\text { release of 4-MU from } \\
\text { 4-MUO (in vitro) }\end{array}$ & [63] \\
\hline & - & - & $115.4 \mu \mathrm{M}$ & - & $\begin{array}{l}\text { release of 4-MU from } \\
\text { 4-MUO (in vitro) }\end{array}$ & [68] \\
\hline & - & - & $13.4 \mu \mathrm{M}$ & - & $\begin{array}{l}\text { release of 4-MU from } \\
\text { 4-MUO (in vitro) }\end{array}$ & [34] \\
\hline
\end{tabular}


Table 1 Continued

\begin{tabular}{|c|c|c|c|c|c|c|}
\hline Active compound & Source & Family & $\begin{array}{l}\mathrm{IC}_{50} \text { (active } \\
\text { compound) }\end{array}$ & $I C_{50}$ (extract) & Test system & Reference(s) \\
\hline $\begin{array}{l}\text { Kaempferol-3-gluco- } \\
\text { side }\end{array}$ & - & - & $70.9 \mu \mathrm{M}$ & - & $\begin{array}{l}\text { release of 4-MU from } \\
\text { 4-MUO (in vitro) }\end{array}$ & {$[68]$} \\
\hline $\begin{array}{l}\text { Kaempferol-3-O- } \beta \text {-d- } \\
\text { glucuronide }\end{array}$ & $\begin{array}{l}\text { Nelumbo nucifera } \\
\text { Gaertn. }\end{array}$ & Nelumbonaceae & $94.00 \mu \mathrm{M}$ & - & $\begin{array}{l}\text { release of 4-MU from } \\
\text { 4-MUO (in vitro) }\end{array}$ & [69] \\
\hline Luteolin & $\begin{array}{l}\text { Chamaecrista } \\
\text { nomame (Sieber) } \\
\text { H.Ohashi }\end{array}$ & Leguminosae & $7.1 \mu \mathrm{M}$ & - & $\begin{array}{l}\text { release of 4-MU from } \\
\text { 4-MUO (in vitro) }\end{array}$ & {$[63]$} \\
\hline Malvidin glykosides & $\begin{array}{l}\text { Vitis rotundifolia } \\
\text { Michx. }\end{array}$ & Vitaceae & - & $\begin{array}{l}11.15 \mathrm{mg} / \mathrm{mL} \\
\text { (MeOH extract) }\end{array}$ & $\begin{array}{l}\text { release of 4-MU from } \\
\text { 4-MUO (in vitro) }\end{array}$ & {$[64]$} \\
\hline \multirow[t]{2}{*}{ Myricetin } & $\begin{array}{l}\text { Intsia palembanica } \\
\text { Miq. }\end{array}$ & Leguminosae & $337.5 \mu \mathrm{M}$ & $\begin{array}{l}4.10 \mu \mathrm{g} / \mathrm{mL} \\
\text { (MeOH extract) }\end{array}$ & $\begin{array}{l}\text { hydrolysis of 2,3-dimer- } \\
\text { capto-1-propanol tribu- } \\
\text { tyrate (in vitro) }\end{array}$ & [43] \\
\hline & - & - & $8.0 \mu \mathrm{M}$ & - & $\begin{array}{l}\text { release of 4-MU from } \\
\text { 4-MUO (in vitro) }\end{array}$ & [63] \\
\hline \multirow[t]{2}{*}{ Neohesperidin } & $\begin{array}{l}\text { Litchi chinensis } \\
\text { Sonn. }\end{array}$ & Sapindaceae & - & $\begin{array}{l}\mathrm{IC}_{44.69}: 7 \mathrm{mg} / \mathrm{mL} \\
\text { (water extract) }\end{array}$ & $\begin{array}{l}\text { release of oleic acid } \\
\text { from triolein (in vitro) }\end{array}$ & [36] \\
\hline & $\begin{array}{l}\text { Citrus reticulata } \\
\text { Blanco }\end{array}$ & Rutaceae & $75.3 \mu \mathrm{M}$ & - & $\begin{array}{l}\text { release of 4-MU from } \\
\text { 4-MUO (in vitro) }\end{array}$ & [53] \\
\hline Orientin & $\begin{array}{l}\text { Eremochloa ophi- } \\
\text { uroides (Munro) } \\
\text { Hack. }\end{array}$ & Poaceae & $31.6 \mu \mathrm{M}$ & - & $\begin{array}{l}\text { hydrolysis of } p \text {-nitro- } \\
\text { phenyl-butyrate } \\
\text { (in vitro) }\end{array}$ & [35] \\
\hline Peonidin & $\begin{array}{l}\text { Vitis rotundifolia } \\
\text { Michx. }\end{array}$ & Vitaceae & - & $\begin{array}{l}11.15 \mathrm{mg} / \mathrm{mL} \\
{[80 \%(\mathrm{v} / \mathrm{v}) \mathrm{MeOH}} \\
\text { extract }]\end{array}$ & $\begin{array}{l}\text { release of 4-MU from } \\
\text { 4-MUO (in vitro) }\end{array}$ & [64] \\
\hline Petunidin & $\begin{array}{l}\text { Vitis rotundifolia } \\
\text { Michx. }\end{array}$ & Vitaceae & - & $\begin{array}{l}11.15 \mathrm{mg} / \mathrm{mL} \\
{[80 \%(\mathrm{v} / \mathrm{v}) \mathrm{MeOH}} \\
\text { extract }]\end{array}$ & $\begin{array}{l}\text { release of 4-MU from } \\
\text { 4-MUO (in vitro) }\end{array}$ & [64] \\
\hline Procyanidin B-2 & $\begin{array}{l}\text { Camellia sinensis } \\
\text { (L.) Kuntze }\end{array}$ & Theaceae & $7.958 \mu \mathrm{M}$ & - & $\begin{array}{l}\text { release of 4-MU from } \\
\text { 4-MUO (in vitro) }\end{array}$ & [39] \\
\hline Procyanidin B-3 & $\begin{array}{l}\text { Camellia sinensis } \\
\text { (L.) Kuntze }\end{array}$ & Theaceae & $2.941 \mu \mathrm{M}$ & - & $\begin{array}{l}\text { release of 4-MU from } \\
\text { 4-MUO (in vitro) }\end{array}$ & [39] \\
\hline $\begin{array}{l}\text { Prodelphinidin A-2 3'- } \\
\text { O-gallate }\end{array}$ & $\begin{array}{l}\text { Camellia sinensis } \\
\text { (L.) Kuntze }\end{array}$ & Theaceae & $0.171 \mu \mathrm{M}$ & - & $\begin{array}{l}\text { release of 4-MU from } \\
\text { 4-MUO (in vitro) }\end{array}$ & [39] \\
\hline Prodelphinidin B-2 & $\begin{array}{l}\text { Camellia sinensis } \\
\text { (L.) Kuntze }\end{array}$ & Theaceae & $2.951 \mu \mathrm{M}$ & - & $\begin{array}{l}\text { release of 4-MU from } \\
\text { 4-MUO (in vitro) }\end{array}$ & [39] \\
\hline $\begin{array}{l}\text { Prodelphinidin B-2 3,3'- } \\
\text { di-O-gallate }\end{array}$ & $\begin{array}{l}\text { Camellia sinensis } \\
\text { (L.) Kuntze }\end{array}$ & Theaceae & $0.107 \mu \mathrm{M}$ & - & $\begin{array}{l}\text { release of 4-MU from } \\
\text { 4-MUO (in vitro) }\end{array}$ & [39] \\
\hline $\begin{array}{l}\text { Prodelphinidin B-2 3'- } \\
\text { O-gallate }\end{array}$ & $\begin{array}{l}\text { Camellia sinensis } \\
\text { (L.) Kuntze }\end{array}$ & Theaceae & $1.969 \mu \mathrm{M}$ & - & $\begin{array}{l}\text { release of 4-MU from } \\
\text { 4-MUO (in vitro) }\end{array}$ & [39] \\
\hline Prodelphinidin B-4 & $\begin{array}{l}\text { Camellia sinensis } \\
\text { (L.) Kuntze }\end{array}$ & Theaceae & $6.230 \mu \mathrm{M}$ & - & $\begin{array}{l}\text { release of 4-MU from } \\
\text { 4-MUO (in vitro) }\end{array}$ & [39] \\
\hline $\begin{array}{l}\text { Prodelphinidin B-4 3'- } \\
\text { O-gallate }\end{array}$ & $\begin{array}{l}\text { Camellia sinensis } \\
\text { (L.) Kuntze }\end{array}$ & Theaceae & $0.223 \mu \mathrm{M}$ & - & $\begin{array}{l}\text { release of 4-MU from } \\
\text { 4-MUO (in vitro) }\end{array}$ & [39] \\
\hline $\begin{array}{l}\text { Prodelphinidin B-5 3,3'- } \\
\text { di-O-gallate }\end{array}$ & $\begin{array}{l}\text { Camellia sinensis } \\
\text { (L.) Kuntze }\end{array}$ & Theaceae & $0.558 \mu \mathrm{M}$ & - & $\begin{array}{l}\text { release of 4-MU from } \\
\text { 4-MUO (in vitro) }\end{array}$ & [39] \\
\hline \multirow[t]{5}{*}{ Quercetin } & $\begin{array}{l}\text { Intsia palembanica } \\
\text { Miq. }\end{array}$ & Leguminosae & $421.1 \mu \mathrm{M}$ & $\begin{array}{l}4.10 \mu \mathrm{g} / \mathrm{mL} \\
\text { (MeOH extract) }\end{array}$ & $\begin{array}{l}\text { hydrolysis of 2,3-dimer- } \\
\text { capto-1-propanol tribu- } \\
\text { tyrate (in vitro) }\end{array}$ & [43] \\
\hline & $\begin{array}{l}\text { Santalum acumina- } \\
\text { tum (R. Br.) A. DC. }\end{array}$ & Santalaceae & - & $\begin{array}{l}0.6 \mathrm{mg} / \mathrm{mL} \\
{[80 \%(\mathrm{v} / \mathrm{v}) \mathrm{MeOH}} \\
\text { extract }]\end{array}$ & $\begin{array}{l}\text { release of 4-MU from } \\
\text { 4-MUO (in vitro) }\end{array}$ & {$[66]$} \\
\hline & - & - & $10 \mu \mathrm{M}$ & - & $\begin{array}{l}\text { release of 4-MU from } \\
\text { 4-MUO (in vitro) }\end{array}$ & {$[63]$} \\
\hline & - & - & $74.6 \mu \mathrm{M}$ & - & $\begin{array}{l}\text { release of 4-MU from } \\
\text { 4-MUO (in vitro) }\end{array}$ & {$[68]$} \\
\hline & - & - & $21.5 \mu \mathrm{M}$ & - & $\begin{array}{l}\text { release of 4-MU from } \\
\text { 4-MUO (in vitro) }\end{array}$ & [34] \\
\hline Quercetin-arabinoside & - & - & $47.9 \mu \mathrm{M}$ & - & $\begin{array}{l}\text { release of 4-MU from } \\
\text { 4-MUO (in vitro) }\end{array}$ & {$[68]$} \\
\hline $\begin{array}{l}\text { Quercetin-3-O- } \beta \text {-D-ara- } \\
\text { binopyranosyl- }(1 \rightarrow 2) \text { - } \\
\beta \text {-D-galactopyranoside }\end{array}$ & $\begin{array}{l}\text { Nelumbo nucifera } \\
\text { Gaertn. }\end{array}$ & Nelumbonaceae & $66.86 \mu \mathrm{M}$ & - & $\begin{array}{l}\text { release of 4-MU from } \\
\text { 4-MUO (in vitro) }\end{array}$ & [69] \\
\hline $\begin{array}{l}\text { Quercetin-3-O- } \beta \text {-D-glu- } \\
\text { curonide }\end{array}$ & $\begin{array}{l}\text { Nelumbo nucifera } \\
\text { Gaertn. }\end{array}$ & Nelumbonaceae & $135.01 \mu \mathrm{M}$ & - & $\begin{array}{l}\text { release of 4-MU from } \\
\text { 4-MUO (in vitro) }\end{array}$ & [69] \\
\hline
\end{tabular}


Table 1 Continued

\begin{tabular}{|c|c|c|c|c|c|c|}
\hline Active compound & Source & Family & $\begin{array}{l}I_{50} \text { (active } \\
\text { compound) }\end{array}$ & $\mathrm{IC}_{50}$ (extract) & Test system & Reference(s) \\
\hline Quercitrin & $\begin{array}{l}\text { Litchi chinensis } \\
\text { Sonn. }\end{array}$ & Sapindaceae & - & $\begin{array}{l}\mathrm{IC}_{44.69}: 7 \mathrm{mg} / \mathrm{mL} \\
\text { (water extract) }\end{array}$ & $\begin{array}{l}\text { release of oleic acid } \\
\text { from triolein (in vitro) }\end{array}$ & {$[36]$} \\
\hline Quercitrin gallate & - & - & $9.0 \mu \mathrm{M}$ & - & $\begin{array}{l}\text { release of 4-MU from } \\
\text { 4-MUO (in vitro) }\end{array}$ & [63] \\
\hline \multirow[t]{3}{*}{ Rutin } & $\begin{array}{l}\text { Litchi chinensis } \\
\text { Sonn. }\end{array}$ & Sapindaceae & - & $\begin{array}{l}\mathrm{IC}_{44.69}: 7 \mathrm{mg} / \mathrm{mL} \\
\text { (water extract) }\end{array}$ & $\begin{array}{l}\text { release of oleic acid } \\
\text { from triolein (in vitro) }\end{array}$ & {$[36]$} \\
\hline & $\begin{array}{l}\text { Capparis sicula } \\
\text { Duhamel }\end{array}$ & Capparaceae & - & - & $\begin{array}{l}\text { hydrolysis of 4-nitro- } \\
\text { phenyl octanoate } \\
\text { (in vitro) }\end{array}$ & [37] \\
\hline & $\begin{array}{l}\text { Eryngium born- } \\
\text { muelleri Nábělek }\end{array}$ & Apiaceae & - & $\begin{array}{l}5.01 \mathrm{mg} / \mathrm{mL} \\
{[80 \%(\mathrm{v} / \mathrm{v}) \text { acetone }} \\
\text { extract] }\end{array}$ & $\begin{array}{l}\text { release of 4-MU from } \\
\text { 4-MUO (in vitro) }\end{array}$ & [70] \\
\hline \multirow[t]{2}{*}{ Theaflavin } & $\begin{array}{l}\text { Camellia sinensis } \\
\text { (L.) Kuntze }\end{array}$ & Theaceae & $0.106 \mu \mathrm{M}$ & - & $\begin{array}{l}\text { release of 4-MU from } \\
\text { 4-MUO (in vitro) }\end{array}$ & [39] \\
\hline & $\begin{array}{l}\text { Camellia sinensis } \\
\text { (L.) Kuntze }\end{array}$ & Theaceae & $1.203 \mu \mathrm{M}$ & - & $\begin{array}{l}\text { release of 4-MU from } \\
4-\mathrm{MUO} \text { (in vitro) }\end{array}$ & {$[40]$} \\
\hline \multirow[t]{2}{*}{$\begin{array}{l}\text { Theaflavin 3,3'-di-O- } \\
\text { gallate }\end{array}$} & $\begin{array}{l}\text { Camellia sinensis } \\
\text { (L.) Kuntze }\end{array}$ & Theaceae & $0.092 \mu \mathrm{M}$ & - & $\begin{array}{l}\text { release of 4-MU from } \\
\text { 4-MUO (in vitro) }\end{array}$ & [39] \\
\hline & $\begin{array}{l}\text { Camellia sinensis } \\
\text { (L.) Kuntze }\end{array}$ & Theaceae & $0.364 \mu \mathrm{M}$ & - & $\begin{array}{l}\text { release of 4-MU from } \\
\text { 4-MUO (in vitro) }\end{array}$ & {$[40]$} \\
\hline \multirow[t]{2}{*}{ Theaflavin 3'-O-gallate } & $\begin{array}{l}\text { Camellia sinensis } \\
\text { (L.) Kuntze }\end{array}$ & Theaceae & $0.112 \mu \mathrm{M}$ & - & $\begin{array}{l}\text { release of 4-MU from } \\
\text { 4-MUO (in vitro) }\end{array}$ & [39] \\
\hline & $\begin{array}{l}\text { Camellia sinensis } \\
\text { (L.) Kuntze }\end{array}$ & Theaceae & $0.447 \mu \mathrm{M}$ & - & $\begin{array}{l}\text { release of 4-MU from } \\
\text { 4-MUO (in vitro) }\end{array}$ & {$[40]$} \\
\hline Theaflavin 3-O-gallate & $\begin{array}{l}\text { Camellia sinensis } \\
\text { (L.) Kuntze }\end{array}$ & Theaceae & $0.514 \mu \mathrm{M}$ & - & $\begin{array}{l}\text { release of 4-MU from } \\
\text { 4-MUO (in vitro) }\end{array}$ & {$[40]$} \\
\hline
\end{tabular}

rutin, quercitrin, neohesperidin, condensed tannins (catechin equivalent), anthocyanins (cyaniding-3-glucoside equivalent) and proanthocyanidins. In addition to the inhibitory potential of the compounds, they were able to reduce epididymal adipose tissue sizes as well as serum and liver lipid content [36].

Zhang et al. studied lipase inhibitory effects of flavonoids from Xanthoceras sorbifolium Bunge (Sapindaceae) [33]. It could be shown that enzyme structure loosens when being promoted by isolated flavonoids [ $45 \%(\mathrm{v} / \mathrm{v})$ ethanol aqueous solution] reducing the affinity of the substrate (olein) for enzyme. Consequently, the activity of the lipase was reduced. Lipase inhibition of $75 \%$ was achieved at a concentration of $0.27 \mathrm{mg} / \mathrm{mL}$ [52].

Lipase inhibitory activities of Mediterranean dietary plants [70\% (v/v) aqueous ethanol extracts] have been reported by Marelli et al. [34]. The highest inhibitory activity with an IC $_{50}$ values of $0.53 \pm 0.03 \mathrm{mg} / \mathrm{mL}$ showed Capparis sicula Duhamel (Capparaceae). Furthermore, the inhibitory potential of Mentha aquatica L. (Lamiaceae, inhibition of $27 \%$ at $1 \mathrm{mg} / \mathrm{mL}$ ), Raphanus raphanistrum L. (Brassicaceae, inhibition of $38 \%$ at $2.5 \mathrm{mg} / \mathrm{mL}$ ) and Echium vulgare $\mathrm{L}$. (Boraginaceae, inhibition of $41 \%$ at $1 \mathrm{mg} / \mathrm{mL}$ ). The lipase inhibitory activity was determined using an in vitro enzyme assay (substrate: 4-nitrophenyl octanoate). The results were attributed to the phenolic constituents of the plants. Particularly the predominance of $( \pm)$ catechin and rutin could be detected [37].

Kawaguchi et al. showed in their in vitro study an inhibiting effect of hesperidin and neohesperidin [53]. 4-MUO was used as a substrate to measure the pancreatic lipase inhibitory activity. It could be demonstrated that the replacement of rutinose (7-position, $\mathrm{R}_{1}$ ) of hesperidin by neohesperidose ( $\bullet$ Fig. 3 ) caused a decrease of the inhibitory activity against porcine PL. Furthermore, hydroxy function in position $3^{\prime}\left(R_{2}\right)$, and methoxy function in po-

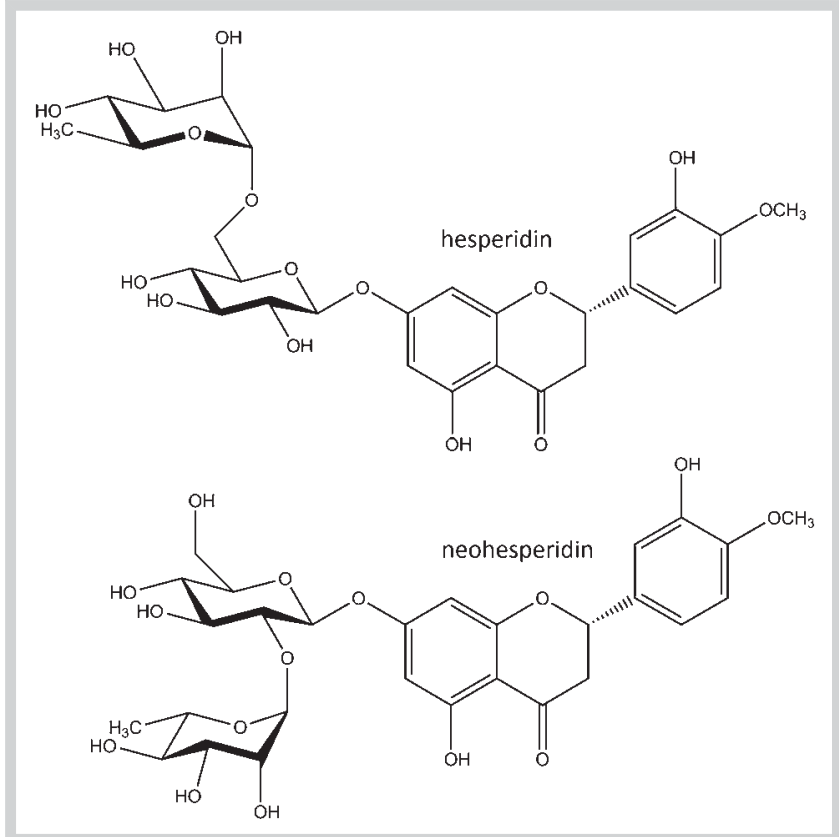

Fig. 3 Chemical structure of hesperidin and neohesperidin.

sition 4' $\left(\mathrm{R}_{3}\right)$ appear to favor inhibition. Eryngium bornmuelleri Náb. (Apiaceae) leaves were investigated for PL inhibition (substrate: 4-MUO, in vitro enzyme assay). Different extracts suppressed enzyme activity in a dose-dependent manner and showed a high phenolic content. Correlation analysis indicated that phenolic compounds were the main sources of the enzyme 
inhibitory activities. Rutin was detected as the major compound (70\% of total phenolics) [38].

A variety of different polyphenols isolated from tea leaves [Camellia sinensis (L.) Kuntze, Theaceae] was evaluated for inhibitory potential against PL inhibition (substrate: 4-MUO, in vitro enzyme assay) by Nakai et al. [39]. Flavan-3-ol digallate esters, such as (-)-epigallo-catechin-3,5-digallate $\left(\mathrm{IC}_{50}: 0.098 \mu \mathrm{M}\right)$, showed a high activity of inhibition on lipase. Furthermore, it was observed that non-esterified flavan-3-ols [(+)-catechin, (-)-epicatechin] showed the lowest inhibitory effect $\left(\mathrm{IC}_{50}:>20 \mu \mathrm{M}\right)$. The results suggest that the presence of galloyl moieties within the structure is responsible for enhancement of PL inhibition. Furthermore it could be demonstrated that the degree of polymerization of flavan-3-ols is important: the inhibitory effect of polymerized polyphenols ( $\mathrm{IC}_{50}: 0.28 \mu \mathrm{g} / \mathrm{mL}$ ) from oolong tea was stronger than that of unfermented and unheated oolong tea $(0.91 \mu \mathrm{g} / \mathrm{mL})$. This hypothesis is supported by the results of Eidenberger et al. [54]. Polyphenols inhibit PL as long as the proportion of condensed polyphenols remains intact. Lipase inhibition is abolished when condensed polyphenols are hydrolysed by acidic treatment. Hotcompressed water (HCW) extracts of black tea (C. sinensis) residues were analyzed for PL inhibiting polyphenols by Yuda et al. [40]. The residues were treated with HCW from 100 to $200^{\circ} \mathrm{C}$. All tested water extracts were able to inhibit PL but extracts obtained at 100 to $140{ }^{\circ} \mathrm{C}$ showed the highest inhibition. Over $150{ }^{\circ} \mathrm{C}$ lipase inhibition was decreased. PL inhibition was determined by measuring the rate of releasing of 4-MU from 4-MUO (in vitro). The positive effect of tea (green, Oolong, black) and tea polyphenols has been confirmed already in human intervention studies [55-57]. Nagao et al. [58] examined the effect of Oolong tea extract with a defined concentration of catechins (690 mg/day) on the body weight of 35 men. After 12 weeks, a significant body weight ( $2.4 \mathrm{~kg}$ decrease), BMI $\left(0.8 \mathrm{~kg} / \mathrm{m}^{2}\right.$ decrease), waist circumference ( $3.4 \mathrm{~cm}$ decrease), and body fat mass ( $1.4 \mathrm{~kg}$ decrease) loss compared with baseline could be determined. Similar results were reported by Tsuchida et al. [59]. 43 male and 37 female participants were treated with a catechin-enriched green tea extract (588 mg catechins/day) for 12 weeks. Body weight ( $1.25 \mathrm{~kg}$ decrease), BMI ( $0.5 \mathrm{~kg} / \mathrm{m}^{2}$ decrease) and body fat mass ( $1.37 \mathrm{~kg}$ decrease) were reduced.

Cocoa (Theobroma cacao L., Malvaceae) extracts, which are rich in proanthocyanins were analyzed by Gu et al. [60]. The results suggest that the degree of polymerization (DP) of proanthocyanins is an important factor in determining inhibitory activity. An inverse correlation between log IC50 and DP was observed. Also in vivo studies showed that proanthocyanidins reduced plasma trigylcerides by inhibiting the absorption of dietary lipids [61,62].

Shimura et al. analyzed the inhibitory effect of different flavonoids on lipase activity (substrate: 4-MUO, in vitro enzyme assay). It was found that a glycosylation of the tested flavonoids caused a decrease of the inhibitory activity. Also flavanones and flavan-3-ols showed negligible activity [63].

You et al. investigated the inhibitory effects of Noble Muscadine grape (Vitis rotundifolia Michx., Vitaceae) extracts. The strongest inhibition on lipase activity (substrate: 4-MUO, in vitro enzyme assay) showed the Noble skin methanolic extract ( $\mathrm{IC}_{50}$ : $11.15 \mathrm{mg} / \mathrm{mL}$ ) and the fruit methanolic extract ( $\mathrm{IC}_{50}: 16.90 \mathrm{mg} /$ $\mathrm{mL}$ ). Using chromatographic and mass spectrometric methods five individual anthocyanins (cyanidin, delphinidin, petunidin, peonidin and malvidin glykosides) could be identified in the Noble muscadine grape extract. As a result, the two anthocyanins cyanidin $\left(\mathrm{IC}_{50}: 591.8 \mu \mathrm{M}\right)$ and cyanidin-3,5-diglucoside ( $\mathrm{IC}_{50}$ :
$1.38 \mathrm{mM}$ ) were tested as references on lipase inhibitory acitivity. The stronger inhibition of cyanidin compared to cyanidin-3,5-diglucoside suggests a higher inhibitory activity of anthocyanins after conversion to aglycone [64].

\section{Phenolic acids}

Phenolic acids are, in addition to the flavonoids, one of the most commonly studied classes of secondary metabolites for lipase inhibition. The basic structure of phenolic acids is formed by hydroxycinnamic and hydroxybenzoic acids. Commonly, they occur as free acids, esters, glycosides or bound complexes. They are widely distributed in plant kingdom and have antioxidant, anticarcinogenic and antimicrobial activity [71]. In recent years it has been demonstrated that some of them have a potential to inhibit PL activity:

Hydroxycinnamic acids are phenolic compounds with major importance for secondary metabolism in plants. They consist of a phenolic ring with a lateral three-carbon chain (C6-C3) (๑ Fig. 2B). Hydroxycinnamic acids and their derivatives are ubiquitously present in fruits and in different parts of plants. Hydroxycinnamic acids are mainly present in form of esters and glycosides. Important examples are $p$-coumaric acid, ferulic acid, caffeic acid and sinapic acid [72-74]. Foods rich in hydroxycinnamic acids are certain types of vegetables (spinach, lettuce, potatoes), fruits (apples, blueberries), coffee and cereals [75].

Identified hydroxycinnamic acids with PL inhibitory potential are shown in Table 2. Gironés-Vilaplana et al. analyzed Citrus sp. fruits (Rutaceae) of Spanish origin (lemon, orange, grapefruit, lime, mandarin) on potential inhibitory activity on PL. Using chromatographic and mass spectrometric methods, the hydromethanolic extract [70\% (v/v) methanol] of Citrus fruits was investigated in more detail. A variety of hydroxycinnamic acid derivatives could be identified: 4-O-coumaroylquinic acid, 3-O-caffeoylferuoylquinic acid, 3-O-feruoylquinic acid hydrated, 3-Ocaffeoylquinic acid, 5-O-caffeoylquinic acid, 5-O-feruoylquinic acid hydrated, ferulic acid, sinapic acid, and 3,5-O-diferuoylquinic acid. In comparison, orange fruit showed the largest amount of hydroxycinnamic acids. However, it is not clear whether the detected cinnamic acids are responsible for the inhibitory effect of the fruits. For the determination of PL activity an in vitro enzymatic assay based on the hydrolysis of natural 1,2-diglyceride was used. Lemon (inhibition of $63.95 \%$ at $0.1 \mathrm{mg} /$ $\mathrm{mL}$ ) and lime (inhibition of $57.17 \%$ at $0.1 \mathrm{mg} / \mathrm{mL}$ ) fruits displayed the highest inhibitory effect on PL. However, no correlation of the detected hydroxycinnamic acids and the inhibitory potential could be determined [76].

Different hydroxycinnamic acids were analyzed by Karama et al. [42]. Ferulic and caffeic acid showed the highest PL inhibitory activity $\left(\mathrm{IC}_{30}: 10 \mu \mathrm{M}\right)$. Inhibition values were determined using $p$ nitrophenyl-acetate as substrate (in vitro). By comparing the results, it is clear that compounds with a methoxy group are weak inhibitors of PL. Also the position of hydroxyl groups in the ring affects lipase inhibition. Inhibitory effects of hydroxyl groups in ortho-position were stronger than at para-position.

Hydroxybenzoic acids are phenolic C6-C1 compounds. They consist of a benzene ring with an added carboxy and hydroxy group as substituent ( $\bullet$ Fig. 2C). They are mainly present in form of esters or glycosides, mostly of vanillic acid, salicylic acid and gentisic acid [55]. Hydroxybenzoic acids are found in common plants such as grapefruit, carrots, onions, grapes and red fruits $[78,79]$. Identified hydroxybenzoic acids with PL inhibitory potential are shown in Table 3. In direct comparison, derivatives of hydroxy- 
Table 2 Comparison of active hydroxycinnamic acids with PL inhibitory activity from different sources. $I C_{50}$ is indicated when available.

\begin{tabular}{|c|c|c|c|c|c|c|}
\hline Active compound & Source & Family & $\begin{array}{l}\mathrm{IC}_{50} \text { (active } \\
\text { compound) }\end{array}$ & $\mathrm{IC}_{50}$ (extract) & Test system & Reference(s) \\
\hline $\begin{array}{l}\text { 2-O-caffeoyl-4-O-gallo- } \\
\text { yl-L-threonic acid }\end{array}$ & $\begin{array}{l}\text { Filipendula camt- } \\
\text { schatica (Pall.) } \\
\text { Maxim. }\end{array}$ & Rosaceae & $26.00 \mu \mathrm{M}$ & - & $\begin{array}{l}\text { release of 4-MU from } \\
\text { 4-MUO (in vitro) }\end{array}$ & {$[65]$} \\
\hline $\begin{array}{l}\text { 3,4-Di-O-caffeoylquinic } \\
\text { acid }\end{array}$ & $\begin{array}{l}\text { Ligularia fischeri } \\
\text { (Ledeb.) Turcz. }\end{array}$ & Compositae & $33.2 \mu \mathrm{M}$ & - & $\begin{array}{l}\text { release of 4-MU from } \\
\text { 4-MUO (in vitro) }\end{array}$ & [41] \\
\hline $\begin{array}{l}\text { 3,5-Di-O-caffeoylquinic } \\
\text { acid }\end{array}$ & $\begin{array}{l}\text { Ligularia fischeri } \\
\text { (Ledeb.) Turcz. }\end{array}$ & Compositae & $40.4 \mu \mathrm{M}$ & - & $\begin{array}{l}\text { release of 4-MU from } \\
\text { 4-MUO (in vitro) }\end{array}$ & {$[41]$} \\
\hline $\begin{array}{l}\text { 3-O-caffeoyl-4-O-gallo- } \\
\text { yl-L-threonic acid }\end{array}$ & $\begin{array}{l}\text { Filipendula camt- } \\
\text { schatica (Pall.) } \\
\text { Maxim. }\end{array}$ & Rosaceae & $246.00 \mu \mathrm{M}$ & - & $\begin{array}{l}\text { release of 4-MU from } \\
\text { 4-MUO (in vitro) }\end{array}$ & {$[65]$} \\
\hline $\begin{array}{l}\text { 4,5-Di-O-caffeoylquinic } \\
\text { acid }\end{array}$ & $\begin{array}{l}\text { Ligularia fischeri } \\
\text { (Ledeb.) Turcz. }\end{array}$ & Compositae & $12.7 \mu \mathrm{M}$ & - & $\begin{array}{l}\text { release of 4-MU from } \\
\text { 4-MUO (in vitro) }\end{array}$ & [41] \\
\hline 4-O-caffeoylquinic acid & $\begin{array}{l}\text { Ligularia fischeri } \\
\text { (Ledeb.) Turcz. }\end{array}$ & Compositae & $253.3 \mu \mathrm{M}$ & - & $\begin{array}{l}\text { release of 4-MU from } \\
\text { 4-MUO (in vitro) }\end{array}$ & {$[41]$} \\
\hline 5-O-caffeoylquinic acid & $\begin{array}{l}\text { Ligularia fischeri } \\
\text { (Ledeb.) Turcz. }\end{array}$ & Compositae & $286.5 \mu \mathrm{M}$ & - & $\begin{array}{l}\text { release of 4-MU from } \\
\text { 4-MUO (in vitro) }\end{array}$ & {$[41]$} \\
\hline 5-O-caffeoylquinic acid & $\begin{array}{l}\text { Ligularia fischeri } \\
\text { (Ledeb.) Turcz. }\end{array}$ & Compositae & $304.8 \mu \mathrm{M}$ & - & $\begin{array}{l}\text { release of 4-MU from } \\
\text { 4-MUO (in vitro) }\end{array}$ & [41] \\
\hline \multirow[t]{4}{*}{ Caffeic acid } & - & - & $\mathrm{IC}_{30}: 10 \mu \mathrm{M}$ & - & $\begin{array}{l}\text { hydrolysis of 4-nitro- } \\
\text { phenyl acetate } \\
\text { (in vitro) }\end{array}$ & {$[42]$} \\
\hline & Echium vulgare $\mathrm{L}$. & Boraginaceae & - & - & $\begin{array}{l}\text { hydrolysis of 4-nitro- } \\
\text { phenyl octanoate (in } \\
\text { vitro) }\end{array}$ & [37] \\
\hline & $\begin{array}{l}\text { Litchichinensis } \\
\text { Sonn. }\end{array}$ & Sapindaceae & - & $\begin{array}{l}\mathrm{IC}_{44.69}: 7 \mathrm{mg} / \mathrm{mL} \\
\text { (water extract) }\end{array}$ & $\begin{array}{l}\text { release of oleic acid } \\
\text { from triolein (in vitro) }\end{array}$ & [36] \\
\hline & $\begin{array}{l}\text { Rosmarinus offici- } \\
\text { nalis L. }\end{array}$ & Lamiaceae & $180.9 \mu \mathrm{M}$ & $\begin{array}{l}13.8 \mu \mathrm{g} / \mathrm{mL} \\
{[80 \%(\mathrm{v} / \mathrm{v}) \mathrm{MeOH}} \\
\text { extract] }\end{array}$ & $\begin{array}{l}\text { hydrolysis of 4-nitro- } \\
\text { phenyl butyrate } \\
\text { (in vitro) }\end{array}$ & [77] \\
\hline Caftaric acid & $\begin{array}{l}\text { Eryngium born- } \\
\text { muelleri Nábělek }\end{array}$ & Apiaceae & - & $\begin{array}{l}5.01 \mathrm{mg} / \mathrm{mL} \\
{[80 \%(\mathrm{v} / \mathrm{v}) \text { acetone }} \\
\text { extract] }\end{array}$ & $\begin{array}{l}\text { release of 4-MU from } \\
\text { 4-MUO (in vitro) }\end{array}$ & [70] \\
\hline \multirow[t]{5}{*}{ Chlorogenic acid } & Echium vulgare $\mathrm{L}$. & Boraginaceae & - & - & $\begin{array}{l}\text { hydrolysis of 4-nitro- } \\
\text { phenyl octanoate (in } \\
\text { vitro) }\end{array}$ & [37] \\
\hline & $\begin{array}{l}\text { Eryngium born- } \\
\text { muelleri Nábělek }\end{array}$ & Apiaceae & - & $\begin{array}{l}5.01 \mathrm{mg} / \mathrm{mL} \\
{[80 \%(\mathrm{v} / \mathrm{v}) \text { acetone }} \\
\text { extract] }\end{array}$ & $\begin{array}{l}\text { release of 4-MU from } \\
\text { 4-MUO (in vitro) }\end{array}$ & {$[70]$} \\
\hline & $\begin{array}{l}\text { Santalum acumina- } \\
\text { tum (R. Br.) A. DC. }\end{array}$ & Santalaceae & - & $\begin{array}{l}0.6 \mathrm{mg} / \mathrm{mL} \\
{[80 \%(\mathrm{v} / \mathrm{v}) \mathrm{MeOH}} \\
\text { extract }]\end{array}$ & $\begin{array}{l}\text { release of 4-MU from } \\
\text { 4-MUO (in vitro) }\end{array}$ & [66] \\
\hline & $\begin{array}{l}\text { Litchi chinensis } \\
\text { Sonn. }\end{array}$ & Sapindaceae & - & $\begin{array}{l}\mathrm{IC}_{44.69}: 7 \mathrm{mg} / \mathrm{mL} \\
\text { (water extract) }\end{array}$ & $\begin{array}{l}\text { release of oleic acid } \\
\text { from triolein (in vitro) }\end{array}$ & [36] \\
\hline & $\begin{array}{l}\text { Rosmarinus offici- } \\
\text { nalis L. }\end{array}$ & Lamiaceae & $272.4 \mu \mathrm{M}$ & $\begin{array}{l}13.8 \mu \mathrm{g} / \mathrm{mL} \\
{[80 \%(\mathrm{v} / \mathrm{v}) \mathrm{MeOH}} \\
\text { extract] }\end{array}$ & $\begin{array}{l}\text { hydrolysis of 4-nitro- } \\
\text { phenyl butyrate } \\
\text { (in vitro) }\end{array}$ & [77] \\
\hline Cinnamic acid & - & - & $\mathrm{IC}_{20}: 10 \mu \mathrm{M}$ & - & $\begin{array}{l}\text { hydrolysis of 4-nitro- } \\
\text { phenyl acetate } \\
\text { (in vitro) }\end{array}$ & {$[42]$} \\
\hline \multirow[t]{4}{*}{ Ferulic acid } & - & - & $\mathrm{IC}_{30}: 10 \mu \mathrm{M}$ & - & $\begin{array}{l}\text { hydrolysis of 4-nitro- } \\
\text { phenyl acetate } \\
\text { (in vitro) }\end{array}$ & {$[42]$} \\
\hline & $\begin{array}{l}\text { Eryngium born- } \\
\text { muelleri Nábělek }\end{array}$ & Apiaceae & - & $\begin{array}{l}5.01 \mathrm{mg} / \mathrm{mL} \\
{[80 \%(\mathrm{v} / \mathrm{v}) \text { acetone }} \\
\text { extract] }\end{array}$ & $\begin{array}{l}\text { release of 4-MU from } \\
\text { 4-MUO (in vitro) }\end{array}$ & [70] \\
\hline & $\begin{array}{l}\text { Litchi chinensis } \\
\text { Sonn. }\end{array}$ & Sapindaceae & - & $\begin{array}{l}\mathrm{IC}_{44.69}: 7 \mathrm{mg} / \mathrm{mL} \\
\text { (water extract) }\end{array}$ & $\begin{array}{l}\text { release of oleic acid } \\
\text { from triolein (in vitro) }\end{array}$ & [36] \\
\hline & - & - & $123.9 \mu \mathrm{M}$ & - & $\begin{array}{l}\text { release of 4-MU from } \\
\text { 4-MUO (in vitro) }\end{array}$ & [34] \\
\hline O-coumaric acid & - & - & $\mathrm{IC}_{20}: 10 \mu \mathrm{M}$ & - & $\begin{array}{l}\text { hydrolysis of 4-nitro- } \\
\text { phenyl acetate } \\
\text { (in vitro) }\end{array}$ & {$[42]$} \\
\hline
\end{tabular}




\begin{tabular}{|c|c|c|c|c|c|c|}
\hline Active compound & Source & Family & $\begin{array}{l}\mathrm{IC}_{50} \text { (active } \\
\text { compound) }\end{array}$ & $\mathrm{IC}_{50}$ (extract) & Test system & Reference(s) \\
\hline \multirow[t]{2}{*}{$P$-coumaric acid } & - & - & $\mathrm{IC}_{17}: 10 \mu \mathrm{M}$ & - & $\begin{array}{l}\text { hydrolysis of 4-nitro- } \\
\text { phenyl acetate } \\
\text { (in vitro) }\end{array}$ & [42] \\
\hline & $\begin{array}{l}\text { Litchi chinensis } \\
\text { Sonn. }\end{array}$ & Sapindaceae & - & $\begin{array}{l}\mathrm{IC}_{44.69}: 7 \mathrm{mg} / \mathrm{mL} \\
\text { (water extract) }\end{array}$ & $\begin{array}{l}\text { release of oleic acid } \\
\text { from triolein (in vitro) }\end{array}$ & [36] \\
\hline \multirow[t]{2}{*}{ Rosmarinic acid } & $\begin{array}{l}\text { Eryngium born- } \\
\text { muelleri Nábělek }\end{array}$ & Apiaceae & - & $\begin{array}{l}5.01 \mathrm{mg} / \mathrm{mL}[ \\
80 \%(\mathrm{v} / \mathrm{v}) \text { acetone } \\
\text { extract] }\end{array}$ & $\begin{array}{l}\text { release of 4-MU from } \\
\text { 4-MUO (in vitro) }\end{array}$ & [70] \\
\hline & $\begin{array}{l}\text { Rosmarinus offici- } \\
\text { nalis L. }\end{array}$ & Lamiaceae & $347.5 \mu \mathrm{M}$ & $\begin{array}{l}13.8 \mu \mathrm{g} / \mathrm{mL} \\
{[80 \%(\mathrm{v} / \mathrm{v}) \mathrm{MeOH}} \\
\text { extract] }\end{array}$ & $\begin{array}{l}\text { hydrolysis of 4-nitro- } \\
\text { phenyl butyrate } \\
\text { (in vitro) }\end{array}$ & [77] \\
\hline \multirow[t]{2}{*}{ Sinapic acid } & - & - & $\mathrm{IC}_{10}: 10 \mu \mathrm{M}$ & - & $\begin{array}{l}\text { hydrolysis of 4-nitro- } \\
\text { phenyl acetate (in vi- } \\
\text { tro) }\end{array}$ & [42] \\
\hline & $\begin{array}{l}\text { Litchichinensis } \\
\text { Sonn. }\end{array}$ & Sapindaceae & - & $\begin{array}{l}\mathrm{IC}_{44.69}: 7 \mathrm{mg} / \mathrm{mL} \\
\text { (water extract) }\end{array}$ & $\begin{array}{l}\text { release of oleic acid } \\
\text { from triolein (in vitro) }\end{array}$ & [36] \\
\hline
\end{tabular}

Table 3 Comparison of active hydroxybenzoic acids with PL inhibitory activity from different sources. $\mathrm{IC}_{50}$ is indicated when available.

\begin{tabular}{|c|c|c|c|c|c|c|}
\hline Active compound & Source & Family & $\begin{array}{l}\mathrm{IC}_{50} \text { (active } \\
\text { compound) }\end{array}$ & $\mathrm{IC}_{50}$ (extract) & Test system & Reference(s) \\
\hline Benzoic acid & - & - & $\mathrm{IC}_{22}: 10 \mu \mathrm{M}$ & - & $\begin{array}{l}\text { hydrolysis of 4-nitro- } \\
\text { phenyl acetate (in vitro) }\end{array}$ & {$[42]$} \\
\hline \multirow[t]{2}{*}{ Gallic acid } & $\begin{array}{l}\text { Litchichinensis } \\
\text { Sonn. }\end{array}$ & Sapindaceae & - & $\begin{array}{l}\mathrm{IC}_{44.69}: 7 \mathrm{mg} / \mathrm{mL} \\
\text { (water extract) }\end{array}$ & $\begin{array}{l}\text { release of oleic acid } \\
\text { from triolein (in vitro) }\end{array}$ & [36] \\
\hline & $\begin{array}{l}\text { Rosmarinus offici- } \\
\text { nalis L. }\end{array}$ & Lamiaceae & $59.4 \mu \mathrm{M}$ & $\begin{array}{l}13.8 \mu \mathrm{g} / \mathrm{mL} \\
(80 \%(\mathrm{v} / \mathrm{v}) \mathrm{MeOH} \\
\text { extract) }\end{array}$ & $\begin{array}{l}\text { hydrolysis of 4-nitro- } \\
\text { phenyl butyrate } \\
\text { (in vitro) }\end{array}$ & [77] \\
\hline \multirow[t]{2}{*}{ Gentisic acid } & - & - & $\mathrm{IC}_{13}: 10 \mu \mathrm{M}$ & - & $\begin{array}{l}\text { hydrolysis of 4-nitro- } \\
\text { phenyl acetate (in vitro) }\end{array}$ & {$[42]$} \\
\hline & $\begin{array}{l}\text { Litchichinensis } \\
\text { Sonn. }\end{array}$ & Sapindaceae & - & $\begin{array}{l}\mathrm{IC}_{44.69:} 7 \mathrm{mg} / \mathrm{mL} \\
\text { (water extract) }\end{array}$ & $\begin{array}{l}\text { release of oleic acid } \\
\text { from triolein (in vitro) }\end{array}$ & {$[36]$} \\
\hline $\begin{array}{l}\text { Hydroxybenzoic acid- } \\
\text { O-hexoside }\end{array}$ & $\begin{array}{l}\text { Eryngium born- } \\
\text { muelleri Nábělek }\end{array}$ & Apiaceae & - & $\begin{array}{l}5.01 \mathrm{mg} / \mathrm{mL} \\
(80 \%(\mathrm{v} / \mathrm{v}) \text { acetone } \\
\text { extract) }\end{array}$ & $\begin{array}{l}\text { release of 4-MU from } \\
\text { 4-MUO (in vitro) }\end{array}$ & [70] \\
\hline$P$-anisic acid & $\begin{array}{l}\text { Litchichinensis } \\
\text { Sonn. }\end{array}$ & Sapindaceae & - & $\begin{array}{l}\mathrm{IC}_{44.69}: 7 \mathrm{mg} / \mathrm{mL} \\
\text { (water extract) }\end{array}$ & $\begin{array}{l}\text { release of oleic acid } \\
\text { from triolein (in vitro) }\end{array}$ & {$[36]$} \\
\hline $\begin{array}{l}\text { P-hydroxybenzoic } \\
\text { acid }\end{array}$ & - & - & $\mathrm{IC}_{21}: 10 \mu \mathrm{M}$ & - & $\begin{array}{l}\text { hydrolysis of 4-nitro- } \\
\text { phenyl acetate (in vitro) }\end{array}$ & {$[42]$} \\
\hline Protocatechuic acid & - & - & $\mathrm{IC}_{12}: 10 \mu \mathrm{M}$ & - & $\begin{array}{l}\text { hydrolysis of 4-nitro- } \\
\text { phenyl acetate (in vitro) }\end{array}$ & {$[42]$} \\
\hline Salicylic acid & - & - & $\mathrm{IC}_{25}: 10 \mu \mathrm{M}$ & - & $\begin{array}{l}\text { hydrolysis of 4-nitro- } \\
\text { phenyl acetate (in vitro) }\end{array}$ & {$[42]$} \\
\hline \multirow[t]{2}{*}{ Syringic acid } & - & - & $\mathrm{IC}_{11}: 10 \mu \mathrm{M}$ & - & $\begin{array}{l}\text { hydrolysis of 4-nitro- } \\
\text { phenyl acetate (in vitro) }\end{array}$ & {$[42]$} \\
\hline & $\begin{array}{l}\text { Litchichinensis } \\
\text { Sonn. }\end{array}$ & Sapindaceae & - & $\begin{array}{l}\mathrm{IC}_{44.69}: 7 \mathrm{mg} / \mathrm{mL} \\
\text { (water extract) }\end{array}$ & $\begin{array}{l}\text { release of oleic acid } \\
\text { from triolein (in vitro) }\end{array}$ & {$[36]$} \\
\hline \multirow[t]{3}{*}{ Vanillic acid } & - & - & $\mathrm{IC}_{17}: 10 \mu \mathrm{M}$ & - & $\begin{array}{l}\text { hydrolysis of 4-nitro- } \\
\text { phenyl acetate (in vitro) }\end{array}$ & {$[42]$} \\
\hline & $\begin{array}{l}\text { Litchi chinensis } \\
\text { Sonn. }\end{array}$ & Sapindaceae & - & - & $\begin{array}{l}\text { release of oleic acid } \\
\text { from triolein (in vitro) }\end{array}$ & {$[36]$} \\
\hline & $\begin{array}{l}\text { Anemarrhena as- } \\
\text { phodeloides Bunge }\end{array}$ & Asparagaceae & - & $\begin{array}{l}\mathrm{IC}_{44.69}: 7 \mathrm{mg} / \mathrm{mL} \\
\text { (water extract) }\end{array}$ & $\begin{array}{l}\text { enzymatic assay } \\
\text { (in vitro) }\end{array}$ & {$[80]$} \\
\hline
\end{tabular}

benzoic acids inhibited less powerfully the activity of PL than hydroxycinnamic acids. Comparing the results with the chemical structure of hydroxybenzoic acids, the influence of methoxy groups in the molecule (less potent) and hydroxyl groups (more potent) can be seen. Furthermore, it could be concluded that a carboxy group takes part in the activity between phenolic acids and the active center of the lipase. Also, the size of the molecule has an influence on a potential lipase inhibition [42]. 
Table 4 Comparison of lignans and their derivatives with PL inhibitory activity from different sources. $\mathrm{IC}_{50}$ is indicated when available.

\begin{tabular}{|c|c|c|c|c|c|c|}
\hline Active compound & Source & Family & $\begin{array}{l}\mathrm{IC}_{50} \text { (active } \\
\text { compound) }\end{array}$ & $I_{50}$ (extract) & Test system & Reference(s) \\
\hline $\begin{array}{l}\text { Dihydrodehydrodico- } \\
\text { niferyl alcohol }\end{array}$ & $\begin{array}{l}\text { Fraxinus rhyncho- } \\
\text { phylla Hance }\end{array}$ & Oleaceae & $\mathrm{IC}_{78}: 278 \mu \mathrm{M}$ & $\begin{array}{l}\mathrm{IC}_{46.1}: 300 \mu \mathrm{gg} / \mathrm{mL} \\
\text { (EtOH fraction) }\end{array}$ & $\begin{array}{l}\text { hydrolysis of 4-nitrophenyl } \\
\text { butyrate (in vitro) }\end{array}$ & [83] \\
\hline Ficusesquilignan & $\begin{array}{l}\text { Fraxinus rhyncho- } \\
\text { phylla Hance }\end{array}$ & Oleaceae & $\mathrm{IC}_{76}: 171 \mu \mathrm{M}$ & $\begin{array}{l}\mathrm{IC}_{46.1}: 300 \mu \mathrm{gg} / \mathrm{mL} \\
\text { (EtOH fraction) }\end{array}$ & $\begin{array}{l}\text { hydrolysis of 4-nitrophenyl } \\
\text { butyrate (in vitro) }\end{array}$ & [83] \\
\hline Hedyotol C & $\begin{array}{l}\text { Fraxinus rhyncho- } \\
\text { phylla Hance }\end{array}$ & Oleaceae & $\mathrm{IC}_{72}: 171 \mu \mathrm{M}$ & $\begin{array}{l}\mathrm{IC}_{46.1}: 300 \mu \mathrm{g} / \mathrm{mL} \\
\text { (EtOH fraction) }\end{array}$ & $\begin{array}{l}\text { hydrolysis of 4-nitrophenyl } \\
\text { butyrate (in vitro) }\end{array}$ & [83] \\
\hline Acernikol & $\begin{array}{l}\text { Fraxinus rhyncho- } \\
\text { phylla Hance }\end{array}$ & Oleaceae & $\mathrm{IC}_{62}: 171 \mu \mathrm{M}$ & $\begin{array}{l}\mathrm{IC}_{46.1}: 300 \mu \mathrm{g} / \mathrm{mL} \\
\text { (EtOH fraction) }\end{array}$ & $\begin{array}{l}\text { hydrolysis of 4-nitrophenyl } \\
\text { butyrate (in vitro) }\end{array}$ & [83] \\
\hline Dihydrobuddlenol B & $\begin{array}{l}\text { Fraxinus rhyncho- } \\
\text { phylla Hance }\end{array}$ & Oleaceae & $\mathrm{IC}_{62}: 171 \mu \mathrm{M}$ & $\begin{array}{l}\mathrm{IC}_{46.1}: 300 \mu \mathrm{g} / \mathrm{mL} \\
\text { (EtOH fraction) }\end{array}$ & $\begin{array}{l}\text { hydrolysis of 4-nitrophenyl } \\
\text { butyrate (in vitro) }\end{array}$ & [83] \\
\hline
\end{tabular}

\section{Lignans}

Lignans are dimers of phenylpropanoid units linked by the central carbons of their side chains (OFig. 2D). In plants, lignans and their higher oligomers act as defensive substances. After oral intake of lignan precursors, they are metabolized to their active form. Lignan precursors are found in a wide variety of plant foods, including flax seeds, sesame seeds, legumes, whole grains (rye, oats, barley), fruits (berries), and vegetables [79,81,82].

Identified lignans and their derivatives with PL inhibitory potential are listed in OTable 4. Isolated compounds from Fraxinus rhynchophylla Hance (Oleaceae) were tested on their inhibitory effects on pancreatic lipase activity by hydrolysis of 4-nitrophenyl butyrate (in vitro). Five isolated compounds (four sesquilignans and one lignan, $\triangle$ Table 4) showed a significant inhibition. Other lignans and coumarinolignans had only weak effects [83].

\section{Conclusion}

$\nabla$

Orlistat is the only pharmacologic agent clinically approved as pancreatic lipase inhibitor in Europe. However, the application of the synthetic drug is associated with a variety of undesirable side effects. Searching for potential inhibitors of PL from natural origin is a current research topic. Previously, a variety of polyphenols were detected as potential active compounds. A structural comparison of known inhibitors makes the following conclusions:

The inhibitory effect of active flavonoids depends on the number and position of phenolic hydroxyl groups. A high number increases the inhibitory effect. Furthermore, non-esterified flavan3 -ols as (+)-catechin and (-)-epicatechin showed a lower inhibition than flavan-3-ol esters. The presence of galloyl moieties within the structure and the polymerization of flavan-3-ols are required for enhancement of PL inhibition. Analysis of flavonoids and anthocyanins suggests a higher inhibitory activity after elimination of glycosylation. Also, the degree of polymerization of proanthocyanins is important. At the investigation of phenolic acids it could be demonstrated that hydroxybenzoic acids inhibit less powerfully the activity of PL than hydroxycinnamic acids. An influence of methoxy groups in the molecule (less potent) and hydroxyl groups (more potent) could be detected. In addition, the size of the molecule and the position of hydroxyl groups in the ring affect lipase inhibition. These results serve as a first basis for finding new natural structures with PL inhibitory activity. By using computational modelling experiments, new potential therapeutic antiobesity agents with less side effects compared to the clinically approved pharmacologic agent Orlistat can be detected. However, further identification and characterization of natural compounds with anti-lipase action is necessary.

\section{Conflict of Interest}

$\nabla$

The authors declare no conflict of interest.

\section{References}

1 WHO. 10 facts on obesity. Geneva: World Health Organization; 2014

2 Kopelman PG. Obesity is a medical problem. Nature 2000; 404: 635643

3 Trigueros L, Peña S, Ugidos AV, Sayas-Barberá E, Pérez-Álvarez JA, Sendra E. Food ingredients as anti-obesity agents: a review. J Food Sci Nutr 2013; 53: 929-942

4 Foster-Schubert KE, Cummings DE. Emerging therapeutic strategies for obesity. Endocr Rev 2006; 27: 779-793

5 Thomson AB, de Pover A, Keelan M, Jarocka-Cyrta E, Clandinin MT. Inhibition of lipid absorption is an approach to the treatment of obesity. Meth Enzymol 1997; 286: 3-41

6 Ros E. Intestinal absorption of triglyceride and cholesterol. Dietary and pharmacological inhibition to reduce cardiovascular risk. Atherosclerosis 2000; 151: 357-379

7 Slanc P, Doljak B, Kreft S, Lunder M, Janes D, Strukelj B. Screening of selected food and medicinal plant extracts for pancreatic lipase inhibition. Phytother Res 2009; 23: 874-877

8 Liu D, Wang F, Tang J, Steglich W, Zhu H. Vibralactone: a lipase inhibitor with an unusual fused beta-lactone produced by cultures of the basidomycete Boreostereum vibrans. Org Lett 2006; 8: 5749-5752

9 Slanc P, Doljak B, Mlinaric A, Strukelj B. Screening of wood damaging fungi and macrofungi for inhibitors of pancreatic lipase. Phytother Res 2004; 18: 758-762

10 Bitou N, Ninomiya M, Tsujita T, Okuda H. Screening of lipase inhibitors from marine algae. Lipids 1999; 34: 441-445

11 Weibel EK, Hadvary P, Hochuli E, Kupfer E, Lengsfeld H. Lipstatin, an inhibitor of pancreatic lipase, produced by Streptomyces toxytricini. I. Producing organism, fermentation, isolation and biological activity. J Antibiot 1987; 40: 1081-1085

12 de la Garza AL, Milagro FI, Boque N, Campión J, Martínez JA. Natural inhibitors of pancreatic lipase as new players in obesity treatment. Planta Med 2011; 77: 773-785

13 Chaput JP, St-Pierre S, Tremblay A. Currently available drugs for the treatment of obesity: Sibutramine and Orlistat. Med Chem 2007; 7: 3-10

14 Bustanji Y, Mohammad M, Hudaib M, Tawaha K, Al-Masri IM, AlKhatib HS, Issa A, Alali FQ. Screening of some medicinal plants for their pancreatic lipase inhibitory potential. Jordan J Pharm Sci 2011; 4: 81-88

15 Gholamhoseinian A, Shahouzehi B, Sharifi-far F. Inhibitory effect of some plant extracts on pancreatic lipase. Int J Pharmacol 2010; 6: 1824

16 Knasmüller S. Krebs und Ernährung. Stuttgart: Georg Thieme Verlag KG; 2014 
17 Field JA, Lettinga G. Toxicity of tannic compounds to microorganisms. In: Hemingway RW, Laks PE, editors. Plant polyphenols: Basic life sciences 59. Heidelberg: Springer Verlag; 1992: 673-692

18 Lambert JD, Sang S, Yang CS. Possible controversy over dietary polyphenols: benefits vs. risks. Chem Res Toxicol 2007; 20: 583-585

19 Roh C, Jung $U$. Screening of crude plant extracts with anti-obesity activity. Int J Mol Sci 2012; 13: 1710-1719

20 Singh S, Jarial R, Kanwar SS. Therapeutic effect of herbal medicines on obesity: herbal pancreatic lipase inhibitors. Wudpecker J Med Plants 2013; 2: 53-65

21 World Health Organization. Obesity and overweight. Factsheet. Geneva: WHO; 2015: 311

22 American Medical Association House of Delegates. Resolution 420 (A-13). Available online: http://www.npr.org/documents/2013/jun/ ama-resolution-obesity.pdf. Accessed: June 11, 2015.

23 Tucci SA, Boyland EJ, Halford JCG. The role of lipid and carbohydrate digestive enzyme inhibitors in the management of obesity: a review of current and emerging therapeutic agents. Diabetes Metab Syndr Obes 2010; 3: 125-143

24 Carey MC, Hernell O. Digestion and absorption of fat. Gastroint Dis 1992; 3: 189-208

25 Winkler FK, D'Arcy A, Hunziker W. Structure of human pancreatic lipase. Nature 1990; 343: 771-774

26 Kimura H, Futami Y, Tarui S, Shinomiya T. Activation of human pancreatic lipase activity by calcium and bile salts. J Biochem 1982; 92: 243251

27 Birari RB, Bhutani KK. Pancreatic lipase inhibitors from natural sources: unexplored potential. Drug Discov Today 2007; 12: 879-889

28 Zeitz VM, Caspary WF, Bockemühl J, Lux G. Ökosystem Darm V. Heidelberg: Springer Verlag; 1993

29 Ebermann R, Elmadfa I. Lehrbuch Lebensmittelchemie und Ernährung. Heidelberg: Springer Verlag; 2011

30 Kuksis A, Shaikh NA, Hoffman AG. Lipid absorption and metabolism. Environ Health Perspect 1979; 33: 45-55

31 Mole S, Waterman PG. Tannic acid and proteolytic enzymes: Enzyme inhibition or substrate deprivation? Phytochemistry 1986; 26: 99-102

32 Griffiths DW. The inhibition of digestive enzymes by extracts of field bean (Vicia faba). J Sci Food Agric 1979; 30: 458-462

33 Haslam E. Plant Polyphenols: Vegetable Tannins revisited. In: Chemistry and Pharmacology of natural Products University of Cambridge Oriental Publications. Cambridge: CUP Archive; 1989

34 Sergent T, Vanderstraeten J, Winand J, Beguin P, Schneider YJ. Phenolic compounds and plant extracts as potential natural anti-obesity substances. Food Chem 2012; 135: 68-73

35 Lee EM, Lee SS, Chung BY, Cho JY, Lee IC, Ahn SR, Jang SJ, Kim TH. Pancreatic lipase inhibition by C-glycosidic flavones isolated from Eremochloa ophiuroides. Molecules 2010; 15: 8251-8259

36 Wu YHS, Chiu CH, Yang DJ, Lin YL, Tseng JK, Chen YC. Inhibitory effects of litchi (Litchi chinensis Sonn.) flower-water extracts on lipase activity and diet-induced obesity. J Funct Foods 2013; 5: 923-929

37 Marrelli M, Loizzo MR, Nicoletti M, Menichini F, Conforti F. In vitro investigation of the potential health benefits of wild Mediterranean dietary plants as anti-obesity agents with $\alpha$-amylase and pancreatic lipase inhibitory activities. J Sci Food Agric 2014; 94: 2217-2224

38 You $Q$, Chen F, Wang X, Jiang Y, Lin S. Anti-diabetic activities of phenolic compounds in muscadine against alpha-glucosidase and pancreatic lipase. LWT - Food Sci Technol 2012; 46: 164-168

39 Nakai M, Fukui Y, Asami S, Toyoda-Ono Y, Iwashita T, Shibata H, Mitsunaga T, Hashimoto F, Kiso Y. Inhibitory effects of oolong tea polyphenols on pancreatic lipase in vitro. J Agric Food Chem 2005; 53: 4593-4598

40 Yuda N, Tanaka M, Suzuki M, Asano Y, Ochi H, Iwatsuki K. Polyphenols extracted from black tea (Camellia sinensis) residue by hot-compressed water and their inhibitory effect on pancreatic lipase in vitro. J Food Sci 2012; 77: H254-H261

41 Cha $\mathrm{KH}$, Song DG, Kim SM, Pan $\mathrm{CH}$. Inhibition of gastrointestinal lipolysis by green tea, coffee, and gomchui (Ligularia fischeri) tea polyphenols during simulated digestion. J Agric Food Chem 2012; 60: 71527157

42 Karamać $M$, Amarowicz $R$. Inhibition of pancreatic lipase by phenolic acids - examination in vitro. Z Naturforsch 1996; 51: 903-905

43 Batubara I, Kuspradini H, Muddathir AM, Mitsunaga T. Intsia palembanica wood extracts and its isolated compounds as Propionibacterium acnes lipase inhibitor. J Wood Sci 2014; 60: 169-174
44 Jeong JY, Jo YH, Lee KY, Do SG, Hwang BY, Lee MK. Optimization of pancreatic lipase inhibition by Cudrania tricuspidata fruits using response surface methodology. Bioorg Med Chem Lett 2014; 24: 2329-2333

45 Yoshikawa M, Shimoda H, Nishida N, Takada M, Matsuda H. Salacia reticulata and its polyphenolic constituents with lipase inhibitory and lipolytic activities have mild antiobesity effects in rats. J Nutr 2002; 132: $1819-1824$

46 Park HJ, Jung UJ, Lee MK, Cho SJ, Jung HK, Hong JH, Park YB, Kim SR, Shim S, Jung J, Choi MS. Modulation of lipid metabolism by polyphenol-rich grape skin extract improves liver steatosis and adiposity in high fat fed mice. Mol Nutr Food Res 2012; 57: 360-364

47 Spencer JPE. Flavonoids: modulators of brain function? Br J Nutr 2008; 99 (E Suppl. 1): ES60-ES77

48 Manach C, Scalbert A, Morand C, Rémésy C, Jiménez L. Polyphenols: food sources and bioavailability. Am J Clin Nutr 2004; 79: 727-747

49 Wollgast J, Anklam E. Review on polyphenols in Theobroma cacao: changes in composition during the manufacture of chocolate and methodology for identification and quantification. Food Res Int 2000; 33: $423-447$

50 Bhagwat S, Haytowitz DB, Holden JM. USDA Database for the Flavonoid Content of Selected Foods $(2001,3)$. Available online: http://www. ars.usda.gov/SP2UserFiles/Place/80400525/Data/Flav/Flav_R03.pdf. Accessed: June 15, 2015.

51 Assini JM, Mulvihill EE, Huff MW. Citrus flavonoids and lipid metabolism. Curr Opin Lipidol 2013; 24: 34-40

52 Zhang HM, Zhou QC, Geng J. Lipase inhibitory effects of flavonoids from Xanthoceras sorbifolia Bunge. World J Pharm Sci 2014; 3: 184-194

53 Kawaguchi K, Mizuno T, Aida K, Uchino K. Hesperidin as an inhibitor of lipases from porcine pancreas and Pseudomonas. Biosci Biotechnol Biochem 1997; 61: 102-104

54 Eidenberger T, Selg M, Fuerst S, Krennhuber K. In-vitro inhibition of human lipase PS by polyphenols from Kiwi fruit. J Food Res 2014; 3: 7177

55 Grove KA, Lambert JD. Laboratory, epidemiological, and human intervention studies show that tea (Camellia sinensis) may be useful in the prevention of obesity. J Nutr 2010; 140: 446-453

56 Wolfram S, Wang $Y$, Thielecke F. Anti-obesity effects of green tea: from bedside to bench. Mol Nutr Food Res 2006; 50: 176-187

57 Huang J, Wang Y, Xie Z, Zhou Y, Zhang Y, Wan X. The anti-obesity effects of green tea in human intervention and basic molecular studies. Eur J Clin Nutr 2014; 68: 1075-1087

58 Nagao T, Komine Y, Soga S, Meguro S, Hase T, Tanaka Y, Tokimitsu I. Ingestion of a tea rich in catechins leads to a reduction in body fat and malondialdehyde-modified LDL in men. Am J Clin Nutr 2005; 81: 122-129

59 Tsuchida $T$, Itakura $H$, Nakamura $H$. Reduction of body fat in humans by long-term ingestion of catechins. Prog Med 2002; 22: 2189-2203

$60 \mathrm{Gu}$ Y, Hurst WJ, Stuart DA, Lambert JD. Inhibition of key digestive enzymes by cocoa extracts and procyanidins. J Agric Food Chem 2011; 59: 5305-5311

61 Quesada H, del Bas JM, Pajuelo D, Díaz S, Fernandez-Larrea J, Pinent M, Arola L, Salvadó MJ, Bladé C. Grape seed proanthocyanidins correct dyslipidemia associated with a high-fat diet in rats and repress genes controlling lipogenesis and VLDL assembling in liver. Int J Obes (Lond) 2009; 33: 1007-1012

62 Bladé C, Arola L, Salvadó MJ. Hypolipidemic effects of proanthocyanidins and their underlying biochemical and molecular mechanisms. Mol Nutr Food Res 2010; 54: 37-59

63 Shimura S, Itoh Y, Yamashita A, Kitano A, Hatano T, Yoshida T, Okuda T. Inhibitory effects of flavonoids on lipase. Nippon Shokuhin Kogyo Gakkaishi 1994; 41: 847-850

64 You $Q$, Chen F, Wang X, Luo PG, Jiang $Y$. Inhibitory effects of muscadine anthocyanins on $\alpha$-glucosidase and pancreatic lipase activities. J Agric Food Chem 2011; 59: 9506-9511

65 Kato E, Yama M, Nakagomi R, Shibata T, Hosokawa K, Kawabata J. Substrate-like water soluble lipase inhibitors from Filipendula kamtschatica. Bioorg Med Chem Lett 2012; 22: 6410-6412

66 Sakulnarmrat K, Srzednicki G, Konczak I. Composition and inhibitory activities towards digestive enzymes of polyphenolic-rich fractions of Davidson's plum and quandong. LWT - Food Sci Technol 2014; 57: 366-375

67 Kumar S, Alagawadi KR. Anti-obesity effects of galangin, a pancreatic lipase inhibitor in cafeteria diet fed female rats. Pharm Biol 2013; 51: 607-613 
68 Zhang B, Deng Z, Ramdath DD, Tang Y, Chen PX, Liu R, Liu Q Tsao R. Phenolic profiles of 20 Canadian lentil cultivars and their contribution to antioxidant activity and inhibitory effects on $\alpha$-glucosidase and pancreatic lipase. Food Chem 2015; 172: 862-872

69 Tao Y, Zhang Y, Wang Y, Cheng Y. Hollow fiber based affinity selection combined with high performance liquid chromatography - mass spectroscopy for rapid screening lipase inhibitors from lotus leaf. Anal Chim Acta 2013; 785: 75-81

70 Dalar A, Türker M, Zabaras D, Konczak I. Phenolic composition, antioxidant and enzyme inhibitory activities of Eryngium bornmuelleri leaf. Plant Foods Hum Nutr 2014; 69: 30-36

71 Kawsar SMA, Huq E, Nahar N, Ozeki Y. Identification and quantification of phenolic acids in Macrotyloma uniflorum by reversed phase HPLC. J Plant Physiol 2008; 3: 165-172

72 Bengoechea L, Hernandez T, Quesada C, Bartolome B, Estrella I, GomezCordoves $C$. Structure of hydroxycinnamic acid derivatives established by high-perfomance liquid chromatography with photodiode-array detection. Chromatographia 1995; 41: 95-98

73 Meyer AS, Donovan JL, Pearson DA, Waterhouse AL, Frankel EN. Fruit hydroxycinnamic acids inhibit human low-density lipoprotein oxidation in vitro. J Agric Food Chem 1998; 46: 1783-1787

74 Herrmann K. Ueber den Gehalt und die Lokalisation der phenolischen Inhaltsstoffe im Gemuese. Qual Plant 1976; 25: 231-246

75 Watzl B, Rechkemmer G. Phenolsäuren. Ernährungs-Umschau 2001; 48: $413-416$
76 Gironés-Vilaplana A, Moreno DA, García-Viguera C. Phytochemistry and biological activity of Spanish Citrus fruits. Food Funct 2014; 5: 764772

77 Bustanji Y, Issa A, Mohammad M, Hudaib M, Tawah K, Alkhatib H, Almasri I, Al-Khalidi B. Inhibition of hormone sensitive lipase and pancreatic lipase by Rosmarinus officinalis extract and selected phenolic constituents. J Med Plant Res 2010; 4: 2235-2242

78 Khadem S, Marles RJ. Monocyclic phenolic acids; hydroxy- and polyhydroxybenzoic acids: Occurrence and recent bioactivity studies. Molecules 2010; 15: 7985-8005

79 Shahidi F, Ho CT. Phenolics in food and natural health products: an overview. Phenolic compounds in foods and natural health products. ACS Symposium Series. Am Chem Soc 2005; 1: 2-8

80 Jo YH, Kim SB, Ahn JH, Liu Q Hwang BY, Lee MK. Inhibitory activity of benzophenones from Anemarrhena asphodeloides on pancreatic lipase. Nat Prod Commun 2013; 8: 481-483

81 Adlercreutz H. Lignans and human health. Crit Rev Clin Lab Sci 2007; 44: 483-525

82 Meagher LP, Beecher GR. Assessment of data on the lignan content of foods. J Food Compos Anal 2000: 13: 935-947

83 Ahn JH, Shin EJ, Liu Q Kim SB, Choi KM, Yoo HS, Hwang BY, Lee MK. Lignan derivatives from Fraxinus rhynchophylla and inhibitory activity on pancreatic lipase. Nat Prod Sci 2012; 18: 116-120 